\title{
Unraveling 1,4-Butanediol Metabolism in Pseudomonas putida KT2440
}

\author{
Wing-Jin Li', Tanja Narancic ${ }^{2,3}$, Shane T. Kenny ${ }^{4}$, Paul-Joachim Niehoff', \\ Kevin O'Connor 2,3, Lars M. Blank' and Nick Wierckx ${ }^{1,5 *}$ \\ ${ }^{1}$ Institute of Applied Microbiology-iAMB, Aachen Biology and Biotechnology-ABBt, RWTH Aachen University, Aachen, \\ Germany, ${ }^{2}$ UCD Earth Institute and School of Biomolecular and Biomedical Science, University College Dublin, Dublin, \\ Ireland, ${ }^{3}$ BEACON - SFI Bioeconomy Research Centre, University College Dublin, Dublin, Ireland, ${ }^{4}$ Bioplastech Ltd., \\ NovaUCD, Belfield Innovation Park, University College Dublin, Dublin, Ireland, ${ }^{5}$ Institute of Bio- and Geosciences IBG-1: \\ Biotechnology, Forschungszentrum Jülich, Jülich, Germany
}

Plastics, in all forms, are a ubiquitous cornerstone of modern civilization. Although humanity undoubtedly benefits from the versatility and durability of plastics, they also cause a tremendous burden for the environment. Bio-upcycling is a promising approach to reduce this burden, especially for polymers that are currently not amenable to mechanical recycling. Wildtype P. putida KT2440 is able to grow on 1,4-butanediol as sole carbon source, but only very slowly. Adaptive laboratory evolution (ALE) led to the isolation of several strains with significantly enhanced growth rate and yield. Genome

OPEN ACCESS

Edited by:

Lei Chen,

Tianjin University, China

Reviewed by:

Shanshan Liu,

The First Affiliated Hospital of Bengbu

Medical College, China

Matilde Fernandez,

University of Granada, Spain

*Correspondence:

Nick Wierckx

n.wierckx@fz-juelich.de

Specialty section:

This article was submitted to

Microbiotechnology,

a section of the journal

Frontiers in Microbiology

Received: 16 December 2019

Accepted: 20 February 2020

Published: 17 March 2020

Citation:

Li W-J, Narancic T, Kenny ST,

Niehoff P-J, O'Connor K, Blank LM

and Wierckx N (2020) Unraveling

1,4-Butanediol Metabolism

in Pseudomonas putida KT2440.

Front. Microbiol. 11:382.

doi: 10.3389/fmicb.2020.00382 re-sequencing and proteomic analysis were applied to characterize the genomic and metabolic basis of efficient 1,4-butanediol metabolism. Initially, 1,4-butanediol is oxidized to 4-hydroxybutyrate, in which the highly expressed dehydrogenase enzymes encoded within the PP_2674-2680 ped gene cluster play an essential role. The resulting 4-hydroxybutyrate can be metabolized through three possible pathways: (i) oxidation to succinate, (ii) CoA activation and subsequent oxidation to succinyl-CoA, and (iii) beta oxidation to glycolyl-CoA and acetyl-CoA. The evolved strains were both mutated in a transcriptional regulator (PP_2046) of an operon encoding both beta-oxidation related genes and an alcohol dehydrogenase. When either the regulator or the alcohol dehydrogenase is deleted, no 1,4-butanediol uptake or growth could be detected. Using a reverse engineering approach, PP_2046 was replaced by a synthetic promotor (14g) to overexpress the downstream operon (PP_2047-2051), thereby enhancing growth on 1,4-butanediol. This work provides a deeper understanding of microbial 1,4-butanediol metabolism in P. putida, which is also expandable to other aliphatic alpha-omega diols. It enables the more efficient metabolism of these diols, thereby enabling biotechnological valorization of plastic monomers in a bio-upcycling approach.

Keywords: laboratory evolution, Pseudomonas putida, proteomics, genomics, plastic upcycling

\section{INTRODUCTION}

Plastics, in all forms, are an ubiquitous cornerstone of modern civilization. They contribute greatly to a more efficient society, i.e., through the reduction of packaging weight, the increase in shelf life of foods, and the insulation of homes and refrigerators. Although humanity undoubtedly benefits from the versatility and durability of plastics, these characteristics also make them a tremendous 
burden for the environment. To reduce this impact, strategies beyond incineration, landfill and inefficient recycling are needed.

One of these approaches involves bio-upcycling, the microbial degradation of plastics and its conversion into value-added material (Wierckx et al., 2015; Narancic and O'Connor, 2017). Proofs of principle for the microbial conversion of selected plastics are already available. For instance, polyethyleneterephthalate (PET) was pyrolized and subsequently converted to polyhydroxyalkanoates (PHA) (Kenny et al., 2008; Kenny et al., 2012). A similar processes enabled conversion of polystyrene and polyethylene to PHA (Ward et al., 2006; Guzik et al., 2014). Polyurethanes (PU) are hardly amenable to mechanical recycling due to their molecular diversity and the fact that many PU are thermosets which can't be molten and re-molded. PU are produced by reacting aliphatic or aromatic diisocyanates with polyols and $\alpha, \omega$-diols as chain extenders. Depending on the monomer composition and chain lengths, polymer properties are diverse, which is key for PU's versatility. Applications can be found in paints and coatings, in building insulation and as sealants, as well as in flexible foams and absorbents for many end-user products like pillows and mattresses. In the context of a bio-upcycling strategy, bacteria and fungi have been found to degrade PU, including several Pseudomonads which grow on PU at high rates (Howard, 2002). A range of $\mathrm{PU}$-degrading ester- and urethane hydrolases have been identified (Hung et al., 2016; Schmidt et al., 2017; Danso et al., 2019; Magnin et al., 2019a,b). Besides this, chemical recycling of $\mathrm{PU}$ is also possible with more mature technologies (Zia et al., 2007; Behrendt and Naber, 2009). In addition to the diamines, which are relatively valuable and can be extracted (Bednarz et al., 2017), typical PU monomers like adipic acid, 1,4-butanediol, and ethylene glycol are released during the process of depolymerization. Degradation pathways for ethylene glycol (Franden et al., 2018; Li et al., 2019) and adipic acid (Parke et al., 2001) are known. Yet, surprisingly little is known about the microbial catabolism of 1,4-butanediol.

1,4-butanediol is one of the major chain extenders used in the production of polyurethanes. It is also a common co-monomer in many polyesters such as polybutylene terephthalate and polybutylene adipate terephthalate. As commodity chemical, 1,4butanediol is used to manufacture 2.5 million tons of plastics and polyesters (Yim et al., 2011). Additionally, it is used as a platform chemical to produce tetrahydrofuran and $\gamma$-butyrolactone, with a total market size valued at USD 6.19 billion in 2015 and is still growing (Grand View Research, 2017). So far, research was mainly focused on the sustainable production of 1,4-butanediol (Burgard et al., 2016). Its de novo microbial production was achieved in $E$. coli by identifying and implementing artificial routes for 1,4-butanediol biosynthesis (Yim et al., 2011). The verified and tested pathway starts with the TCA cycle intermediate succinyl-CoA. The heterologous CoA-dependent succinate semialdehyde dehydrogenase (SucD) from Clostridium kluyveri and either a native or heterologous 4-hydroxybutyrate dehydrogenase from C. kluyveri, Porphyromonas gingivalis or Ralstonia eutropha catalyze the reaction from succinyl-CoA to 4-hydroxybutyrate. After CoA activation, 4-hydroxybutyryl-CoA will be further reduced by alcohol and aldehyde dehydrogenases to the final product 1,4-butanediol. In addition to this commonly used pathway, alternative potential routes via $\alpha$-ketoglutarate, glutamate or acetyl-CoA were described (Yim et al., 2011). Conversion of xylose to 1,4-butanediol has also been described (Liu and Lu, 2015).

Butanol is a substrate with structural similarities to 1,4butanediol. Usually, butanol concentrations above 1-2\% (135$270 \mathrm{mM}$ ) are toxic or at least growth-inhibiting for most of microbes, including Pseudomonas putida BIRD-1, DOT-T1E, and KT2440 (Cuenca et al., 2016). Nevertheless, Pseudomonas exhibits promising traits on tolerating, assimilating or at least surviving butanol (Rühl et al., 2009). To cope with butanol, classic solvent defense mechanisms like efflux pumps, membrane modifications or rebalancing of the redox state are activated (Ramos et al., 2002; Basler et al., 2018). Further, P. putida KT2440 is capable of rapid butanol oxidation to butyrate via a variety of alcohol- and aldehyde dehydrogenases (Simon et al., 2015; Vallon et al., 2015; Cuenca et al., 2016). Prominent among these are PedE, PedH, and PedI alcohol and aldehyde dehydrogenases, encoded in the so-called ped cluster. These have a highly relaxed substrate specificity and are capable of oxidizing, among others, ethanol, phenylethanol, butanol, and butanal (butyraldehyde) (Wehrmann et al., 2017). The resulting butyrate is CoA-activated by acyl-CoA synthetases like AcsA1 (PP_4487), and subsequently undergoes $\beta$-oxidation.

Non-pathogenic Pseudomonads have an established track record in bioremediation and biodegradation processes (Samanta et al., 2002; Spini et al., 2018; Tahseen et al., 2019), and different strains of this genus are also suitable candidates to perform bio-upcycling (Kenny et al., 2008; Wierckx et al., 2015; Wilkes and Aristilde, 2017). One of the widely used biotechnological hosts is $P$. putida KT2440, which possesses extensive metabolic abilities (Nelson et al., 2002; Nikel et al., 2014; Nikel and de Lorenzo, 2018). Being a soil bacterium and therefore exposed to different environmental surroundings, it is equipped with tolerances and metabolic capabilities toward a broad spectrum of substances. The $6.18 \mathrm{Mb}$ genome of $P$. putida KT2440 harbors a broad spectrum of oxygenases, oxidoreductases as well as hydrolases, transferases, and dehydrogenases (Belda et al., 2016). This wide range of enzymes enables $P$. putida KT2440 to modify an abundance of alcohols and aldehydes (Wierckx et al., 2011).

In this work, $P$. putida KT2440 strains with an enhanced growth rate on 1,4-butanediol are obtained by adaptive laboratory evolution (ALE), and analyzed by proteomics and genome resequencing in order to determine possible degradation routes. The improved growth phenotype was subsequently reverse-engineered into the wildtype, thereby generating a deeper understanding of 1,4-butanediol metabolism and broadening the applicability of $P$. putida for plastic upcycling.

\section{MATERIALS AND METHODS}

\section{Chemicals, Media, and Cultivation Conditions}

The chemicals used in this work were obtained from Carl Roth (Karlsruhe, Germany), Sigma-Aldrich (St. Louis, MO, 
United States), or Merck (Darmstadt, Germany) unless stated otherwise. Glycerol was kindly provided by Bioeton (Kyritz, Germany).

All strains used in this work are listed in Table 1. Cultivations were performed in $\mathrm{LB}$ - complex medium $\left(10 \mathrm{~g} \mathrm{~L}^{-1}\right.$ tryptone, $5 \mathrm{~g} \mathrm{~L}^{-1}$ yeast extract and $5 \mathrm{~g} \mathrm{~L}^{-1}$ sodium chloride) or, for quantitative microbiology experiments, in mineral salt medium (MSM) (Hartmans et al., 1989), solidified when needed with $1.5 \%$ agar $(\mathrm{w} / \mathrm{v})$, containing different amount of $\mathrm{C}$ source. Precultures were supplied with $20 \mathrm{mM}$ glucose, whereas $20 \mathrm{mM}$ 1,4-butanediol were used for studies with 1,4-butanediol.

For plasmid maintenance, E. coli strains and P. putida KT2440 strains were cultivated in media supplemented with $50 \mathrm{mg} \mathrm{L}^{-1}$ kanamycin, which was sterilized by using a $0.2 \mu \mathrm{m}$ syringe filter (Carl Roth GmbH + Co. KG, Karlsruhe, Germany).

Liquid cultivations were incubated at $30^{\circ} \mathrm{C}$ for Pseudomonas and $37^{\circ} \mathrm{C}$ for E. coli, $200 \mathrm{rpm}$ shaking speed with an amplitude of $50 \mathrm{~mm}$ in a Multitron shaker (INFORS, Bottmingen, Switzerland) using $100 \mathrm{~mL}$ non-baffled Erlenmeyer flasks with metal caps, containing $10 \mathrm{~mL}$ culture volume for a pre-culture and $500 \mathrm{~mL}$ non-baffled Erlenmeyer flasks with metal caps, containing $50 \mathrm{~mL}$ culture volume for a main culture.

For online growth detection, 96-well plates with $200 \mu \mathrm{L}$ or 24-well plates with 4-3 mL culture volume were inoculated with a pre-culture containing 4-3 $\mathrm{mL}$ MSM with $20 \mathrm{mM}$ glucose in 24-well System Duetz plates (Enzyscreen, Heemstede, The Netherlands), cultivated in a Multitron shaker (INFORS, Bottmingen, Switzerland) with a $300 \mathrm{rpm}$ shaking speed with an amplitude of $50 \mathrm{~mm}$. Inoculated Growth Profiler ${ }^{\circledR}$ plates were incubated at $30^{\circ} \mathrm{C}, 225 \mathrm{rpm}$ shaking speed with an amplitude of $50 \mathrm{~mm}$ in the Growth Profiler ${ }^{\circledR} 960$ (Enzyscreen, Heemstede, The Netherlands).

Adaptive laboratory evolution (ALE) was performed as follows: a pre-culture of $P$. putida KT2440, cultivated in MSM with $20 \mathrm{mM}$ glucose, was used to inoculate $250 \mathrm{~mL}$ clear glass Boston bottles with Mininert valves (Thermo Fisher Scientific, Waltham, MA, United States) containing $20 \mathrm{mM} \mathrm{1,4-}$ butanediol for the adaptation on 1,4-butanediol (final $\mathrm{OD}_{600}$ of 0.01). Serial transfers were reinoculated several times after the cultures reached an $\mathrm{OD}_{600}$ of at least 0.5 , with a starting $\mathrm{OD}_{600}$ of 0.1 . After growth was detected (usually overnight), single colonies were isolated from ALE cultures by streaking samples on LB agar plates. After ALE on 1,4-butanediol, two strains (B10.1 and B10.2) out of 72 strains were selected according to their growth behavior in MSM with $20 \mathrm{mM}$ 1,4-butanediol determined using the Growth Profiler ${ }^{\circledR} 960$ (Enzyscreen, Heemstede, The Netherlands).

Growth experiments for PHA accumulation were carried out in $250 \mathrm{~mL}$ Erlenmeyer flasks containing $50 \mathrm{~mL}$ of nitrogen limited MSM medium supplemented with $80 \mathrm{mM} \mathrm{1,4}$ butanediol. Nitrogen limited MSM medium contains $9 \mathrm{~g} / \mathrm{L} \mathrm{Na}_{2} \mathrm{HPO}_{4} \cdot 12 \mathrm{H}_{2} 0$, $1.5 \mathrm{~g} / \mathrm{L} \mathrm{KH}_{2} \mathrm{PO}_{4}, 0.25 \mathrm{~g} / \mathrm{N} \mathrm{N}_{4} \mathrm{Cl}$, trace elements. An overnight culture was prepared by inoculating $2 \mathrm{~mL}$ of medium with a single colony from a plate and incubating overnight at $30^{\circ} \mathrm{C}$ and shaking at $200 \mathrm{rpm}$. Five hundred micro liter of the $2 \mathrm{~mL}$ MSM overnight culture was used as an inoculum for the $50 \mathrm{~mL}$ cultures which were incubated under the same conditions for $48 \mathrm{~h}$. Octanoic acid $(20 \mathrm{mM})$ was added to some flasks after $24 \mathrm{~h}$.

\section{Molecular Work DNA Procedures}

The construction of plasmids was performed either by standard restriction-ligation or Gibson assembly (Gibson et al., 2009) using the NEBuilder HiFi DNA Assembly (New England Biolabs, Ipswich, MA, United States). DNA modifying enzymes were purchased from New England Biolabs, for dephosphorylation Fast AP Thermo Sensitive Alkaline Phosphatase (Thermo Fisher Scientific, Langenselbold, Germany) was used. Primers were purchased as unmodified DNA oligonucleotides from Eurofins Genomics (Ebersberg, Germany) and are listed in Supplementary Table S1. Clonal DNA sequences were amplified using the Q5 High-Fidelity Polymerase (New England Biolabs, Ipswich, MA, United States). DNA- ligations were performed by using T4 ligase from Fermentas (Thermo Fisher Scientific, Langenselbold, Germany) according to the protocol. Arbitraryprimed PCR was performed as described by Martínez-García et al. (2014). For the transformation of DNA assemblies and purified plasmids (Supplementary Table S2) into competent E. coli a heat shock protocol was performed (Hanahan, 1983). For $P$. putida transformations either conjugational transfer or electroporation were performed as described by Wynands et al. (2018). Knockout strains were obtained using the pEMG system described by Martínez-García and de Lorenzo (2011) with a modified protocol described by Wynands et al. (2018). Plasmid inserts and gene deletions were confirmed by Sanger sequencing performed by Eurofins Genomics (Ebersberg, Germany).

In order to perform PCR directly from bacteria the alkaline polyethylene glycerol-based method was used (Chomczynski and Rymaszewski, 2006). Therefore, cell material was picked and dissolved in $50 \mu \mathrm{L}$ of the reagent, containing $60 \mathrm{~g}$ PEG 200 with $0.93 \mathrm{~mL} 2 \mathrm{M} \mathrm{KOH}$ and $39 \mathrm{~mL}$ water, with a $\mathrm{pH}$ of 13.4. After incubation for 3-15 $\mathrm{min}, 2 \mu \mathrm{L}$ of the sample was used as template in a $25 \mu \mathrm{L}$ PCR reaction.

\section{Analytical Methods \\ Growth Monitoring Methods}

Bacterial growth was monitored as optical density at a wavelength of $\lambda=600 \mathrm{~nm}\left(\mathrm{OD}_{600}\right)$ with an Ultrospec 10 Cell Density Meter (GE Healthcare, Little Chalfont, Buckinghamshire, United Kingdom). Growth rates $(\mu)$ are determined by fitting an exponential curve to a plot of $\mathrm{OD}_{600}$ over time of a culture in the exponential phase. The online analysis of growth using the Growth Profiler ${ }^{\circledR}$ was analyzed using the Growth Profiler ${ }^{\circledR}$ Control software V2_0_0. Cell densities are expressed as G-value, which is derived from imaging analysis of microtiter plates with transparent bottoms.

\section{PHA Analysis}

Cells were harvested by centrifugation at $3320 \times g$ for $10 \mathrm{~min}$ and then lyophilized and weighed for determination of cell dry weight. PHA content was determined by subjecting lyophilized cells to acidic methanolysis (Brandl et al., 1988; Lageveen et al., 1988). Five to ten milligram of dried cells were resuspended 
TABLE 1 | Pseudomonas putida strains used in this work with listed genotype and references.

\begin{tabular}{|c|c|c|c|}
\hline No. & Strain & Genotype & References \\
\hline 1 & KT2440 wildtype & Cured, restriction-deficient derivative of $P$. putida mt-2 & Bagdasarian et al., 1981 \\
\hline 2 & B10.1 & KT2440 ALE in BDO, single strain A6 & This work \\
\hline 3 & B10.2 & KT2440 ALE in BDO, single strain C2 & This work \\
\hline \multirow[t]{3}{*}{4} & KT2440 $\Delta$ PP_2046 & $\Delta P P \_2046$ in KT2440 & This work \\
\hline & B10.1 APP_2046 & $\Delta$ PP_2046 in B10.1 & This work \\
\hline & B10.2 $\Delta$ PP_2046 & APP_2046 in B10.2 & This work \\
\hline \multirow[t]{3}{*}{5} & KT2440 APP_2046:14g & $\Delta$ PP_2046:14g in KT2440 & This work \\
\hline & B10.1 $\Delta$ PP_2046:14g & $\Delta$ PP_2046:14g in B10.1 & This work \\
\hline & B10.2 $\Delta$ PP_2046:14g & $\Delta P P \_2046: 14 g$ in B10.2 & This work \\
\hline 6 & KT2440 $\Delta$ ped & $\Delta$ pedE-I in KT2440 & Li et al., 2019 \\
\hline \multirow[t]{2}{*}{7} & KT2440 $\Delta$ pedE & $\Delta$ pedE in KT2440 & This work \\
\hline & KT2440 $\Delta$ pedH & $\Delta$ pedH in $\mathrm{KT} 2440$ & This work \\
\hline 8 & KT2440 $\Delta$ pedl & $\Delta$ pedl in KT2440 & This work \\
\hline \multirow[t]{3}{*}{9} & KT2440 APP_2047-2051 & knockout PP_2047-51 in KT2440 & This work \\
\hline & B10.A SPP_2047-2051 & knockout PP_2047-51 in B10.A & This work \\
\hline & B10.B APP_2047-2051 & knockout PP_2047-51 in B10.B & This work \\
\hline \multirow[t]{3}{*}{10} & KT2440 $\Delta$ PP_2049 & $\Delta \mathrm{PP} \_2049$ in KT2440 & This work \\
\hline & B10.1 $\Delta$ PP_2049 & $\Delta \mathrm{PP} \_2049$ in B10.1 & This work \\
\hline & B10.2 APP_2049 & APP_2049 in B10.2 & This work \\
\hline \multirow[t]{6}{*}{11} & 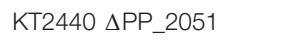 & $\Delta P P \_2051$ in KT2440 & This work \\
\hline & B10.1 APP_2051 & APP_2051 in B10.1 & This work \\
\hline & B102 $\Delta$ PP_2051 & $\Delta \mathrm{PP} \_2051 \mathrm{in} \mathrm{B} 10.2$ & This work \\
\hline & KT2440 $\Delta$ PP_0411-13 & $\Delta P P_{-} 0411-13$ & This work \\
\hline & B10.1 $\Delta$ PP_0411-13 & $\Delta$ PP_0411-13 & This work \\
\hline & B10.2 $\Delta$ PP_0411-13 & $\Delta$ PP_0411-13 & This work \\
\hline
\end{tabular}

in $2 \mathrm{~mL}$ of acidified methanol $\left(15 \% \mathrm{H}_{2} \mathrm{SO}_{4}, \mathrm{v} / \mathrm{v}\right)$ and $2 \mathrm{~mL}$ of chloroform containing $6 \mathrm{mg} / \mathrm{l}$ benzoate methyl ester as an internal standard. The solution was placed in $15 \mathrm{~mL}$ Pyrex test tubes, sealed and incubated at $100^{\circ} \mathrm{C}$ for $3 \mathrm{~h}$. The tubes were then placed on ice for $1 \mathrm{~min}$. One milliliter of water was added to each tube and the solution mixed by vigorous vortexing. The phases were allowed to separate, and the organic phase was removed and passed through a filter before further analysis.

The 3-hydroxyalkanoic acid methyl esters were analyzed by gas chromatography (GC) using an Agilent $6890 \mathrm{~N}$ chromatograph equipped with a HP Innowax column (30 $\mathrm{m} \times 0.25 \mathrm{~mm} \times 0.5 \mu \mathrm{m})$ and a flame ionization detector (FID). An oven ramp cycle was employed as follows, $120^{\circ} \mathrm{C}$ for $5 \mathrm{~min}$, increasing by $3^{\circ} \mathrm{C} / \mathrm{min}$ to $180^{\circ} \mathrm{C}, 180^{\circ} \mathrm{C}$ for $10 \mathrm{~min}$. A 20:1 split was used with helium as the carrier gas and an inlet temperature of $250^{\circ} \mathrm{C}$. Commercially available 3hydroxyalkanoic acids (Bioplastech Ltd., Dublin, Ireland) were methylated as described above for PHA samples and used as standards to identify PHA monomers.

\section{Extracellular Metabolites}

For measuring extracellular metabolites, samples taken from liquid cultivation were centrifuged for $3 \mathrm{~min}$ at $17,000 \times g$ to obtain supernatant for High-Performance Liquid Chromatography (HLPC) analysis using a Beckman System Gold 126 Solvent Module equipped with a Smartline 2300 refractive index detector (Knauer, Berlin, Germany). Analytes were eluted using a $300 \times 8 \mathrm{~mm}$ organic acid resin column together with a $40 \times 8 \mathrm{~mm}$ organic acid resin precolumn (both from CS Chromatographie, Langerwehe, Germany) with $5 \mathrm{mM} \mathrm{H}_{2} \mathrm{SO}_{4}$ as mobile phase at a flow rate of $0.7 \mathrm{~mL} \mathrm{~min}^{-1}$ at $70^{\circ} \mathrm{C}$ (Li et al., 2019).

\section{Genome Sequencing}

Genomic DNA for resequencing was isolated through a High Pure PCR Template Preparation Kit (ROCHE life science, Basel, Switzerland). Sequencing and SNP/InDel (single nucleotide polymorphism/insertion and deletion polymorphism) calling was done by GATC (Konstanz, Germany) using Illumina technology as paired-end reads of 125 base pairs. To map the reference sequence against the database, BWA with default parameters was used (Li and Durbin, 2009). SNPs and InDels, analyzed by GATK's UnifiedGenotyper (McKenna et al., 2010; DePristo et al., 2011), were listed and visualized with the Integrative Genomics Viewer (IGV) (Thorvaldsdóttir et al., 2013).

The sequences have been deposited in the NCBI Sequence Read Archive (SRA) with the accession number SRP148839 for ethylene glycol ALE strains (including our laboratory wildtype SRX4119395 used in this study) and SRP148839 for the 1,4butanediol ALE strains.

\section{Proteomics}

The evolved strains B10.1 and B10.2 were cultivated along with the wild type $P$. putida KT2440 in $50 \mathrm{~mL}$ MSM medium supplemented with $20 \mathrm{mM}$ 1,4-butanediol or $13 \mathrm{mM}$ glucose (both equivalent to $80 \mathrm{mM} \mathrm{C}$ ). The cultures were harvested 
by centrifugation and prepared for proteomic analysis as previously described (Narancic et al., 2016). Samples were sent to T. Narancic at University of Dublin to perform the following protocol. For total protein concentrations, peptide fragments obtained by trypsin digestion were analyzed on the Q-Exactive Hybrid Quadrupole Orbitrap Mass Spectrometer (MS; Thermo Fisher Scientific) connected to a Dionex Ultimate 3000 (RSLCnano; Thermo Fisher Scientific) chromatography system (Buffer A: 97\% water, 2.5\% acetonitrile, 0.5\% acetic acid; buffer B: $97 \%$ acetonitrile, $2.5 \%$ water, $0.5 \%$ acetic acid; all solvents were LC-MS grade). The mass spectrometer was operated in positive ion mode with a capillary temperature of $320^{\circ} \mathrm{C}$ and a potential of $2300 \mathrm{~V}$ applied to the frit. All data were acquired with the MS operating in automatic datadependent switching mode. A high-resolution (70,000) MS scan (300-1600 m/z) was performed using the Q Exactive to select the 12 most intense ions prior to MS/MS analysis using HCD. The identification and quantification were performed using the Andromeda peptide identification algorithm integrated into MaxQuant (Cox and Mann, 2008; Cox et al., 2011). P. putida KT2440 protein sequence database downloaded from UniProt ${ }^{1}$ in April 2016 was used as a reference (The UniProt Consortium, 2019). Label-free quantification (LFQ) was used to compare the expression level of proteins across samples and growth conditions (Wang et al., 2003). Proteins with a twofold change or higher and a significant change in $t$-test (FDR 0.01) were automatically accepted, while spectra with no specific change were manually checked for quality.

Each sample had three biological replicates, and each biological replicate was then prepared for the proteomic analysis as a technical replicate. Statistical analysis was performed using Perseus and built-in Welche's $t$-test with FDR set at 0.01 (Tyanova et al., 2016). The proteins with at least twofold change were functionally annotated using David bioinformatics (Huang et al., 2009a,b) and clustered into orthologous groups using EggNOG (Huerta-Cepas et al., 2016).

\section{Detection of Dehydrogenase Activity}

To perform the enzyme assay, cells from a pre-culture were used to inoculate the main culture containing $20 \mathrm{mM}$ glucose and $5 \mathrm{mM}$ 1,4-butanediol. After $16 \mathrm{~h}$ of cultivation, crude extract was isolated using BugBuster (Merck, Darmstadt, Germany) and was desalted using PD-desalting columns (GE Healthcare, Buckinghamshire, United Kingdom) and eluted in $100 \mathrm{mM}$ glycinglycin buffer. Protein concentrations were estimated by standard Bradford test at $595 \mathrm{~nm}$. For the dehydrogenase assay, a modified protocol from Kagi and Vallee (1960) was followed, in which $5 \mathrm{mM} 4$-hydroxybutyrate or $4 \%$ ethanol as control were used as substrate. The formation of $\mathrm{NADH}$ was measured at $340 \mathrm{~nm}$ in a 96 -well-plate at $30^{\circ} \mathrm{C}$ in a well plate reader from Synergy Mx from Biotek (Bad Friedrichshall, Germany). To obtain a homogeneous mixture, after the addition of $\mathrm{NAD}^{+}$or 4-hydroxybutyrate, the well-plate was shaken for $3 \mathrm{~s}$ at highest speed available.

\footnotetext{
${ }^{1}$ www.uniprot.org
}

\section{Statistics}

Statistical probability values were, if not stated otherwise, calculated using a paired Student's t-distribution test with homogeneity of variance ( $n=3$, significance level of 0.05$)$. In case of duplicates, errors are expressed as deviation from the mean $(n=2)$.

\section{RESULTS}

\section{Isolation of Strain With Enhanced Growth on 1,4-Butanediol by ALE}

To study the growth of $P$. putida KT2440 and possible intermediate production when metabolizing 1,4-butanediol, growth experiments in shake flasks were performed. The wildtype showed poor growth $\left(\mu_{\max }=0.082 \pm 0.004 \mathrm{~h}^{-1}\right)$ on MSM with $20 \mathrm{mM}$ 1,4-butanediol, requiring more than $50 \mathrm{~h}$ to consume all substrate $(t=49 ; 1.3 \pm 0.1 \mathrm{mM})$, while secreting high levels of the oxidation product 4-hydroxybutyrate $(t=49 \mathrm{~h}$; $16.8 \pm 0.3 \mathrm{mM}$ ) (Figure 1). This slow growth implies that in principle the metabolic routes are present in P. putida KT2440, but they are not operating optimally under the chosen conditions.

To enhance its ability to grow on 1,4-butanediol, wildtype P. putida KT2440 was subjected to ALE. This method is known to enable the selection of mutated strains with enhanced properties toward specific environments, likely affecting transcriptional regulatory systems (Dragosits and Mattanovich, 2013; Lennen et al., 2019; Li et al., 2019). Cultures of P. putida KT2440 were serially re-inoculated to fresh media containing $20 \mathrm{mM} \mathrm{1,4-}$ butanediol ten times, as soon as growth was observed in form of optical densities above 0.8 (Figure 1). All three parallel evolution lines grew with the same trend. While the first three batches reached an $\mathrm{OD}_{600}$ between 0.8 and 2 after 3-4 days, later batches reached an $\mathrm{OD}_{600}$ between 2.5 and 3.5 after 2 days or less. The ALE was stopped after approximately 47 generations, when growth on 1,4-butanediol reached an $\mathrm{OD}_{600}$ of 2.5 overnight. Single colonies were isolated by streaking the three ALE cultures on LB plates. From each of the three evolution lines, 24 single colonies were tested for growth on 1,4-butanediol in a 96- well plate using a Growth Profiler ${ }^{\circledR}$ (Figure 1). Overall, the growth of these single clones was relatively similar, indicating a rather homogeneous evolved population. The two strains with the highest growth rates were selected, from different evolutionary lines, according to their growth in MSM with $20 \mathrm{mM} \mathrm{1,4-}$ butanediol. These isolated clonal strains are named B10.1 and B10.2 (Figure 1).

The two evolved strains grew faster on 1,4-butanediol than the wildtype, even after several generations in complex medium or MSM containing glucose, indicating that the observed phenotype was evolutionary fixed in the genome. The single evolved isolates B10.1 and B10.2 reach growth rates of $0.33 \pm 0.054 \mathrm{~h}^{-1}$ and $0.31 \pm 0.001 \mathrm{~h}^{-1}$, respectively. In contrast to the wildtype they completely consume all carbon source, reaching maximum biomass concentrations of $1.05 \pm 0.0 \mathrm{~g}_{c d w} \mathrm{~L}^{-1}$ and $0.96 \pm 0.02$ $\mathrm{g}_{c d w} \mathrm{~L}^{-1}$ after $33 \mathrm{~h}$. This translates to an average biomass yield of $0.56 \pm 0.025 \mathrm{~g} / \mathrm{g}$ for the evolved strains. This relatively high yield 


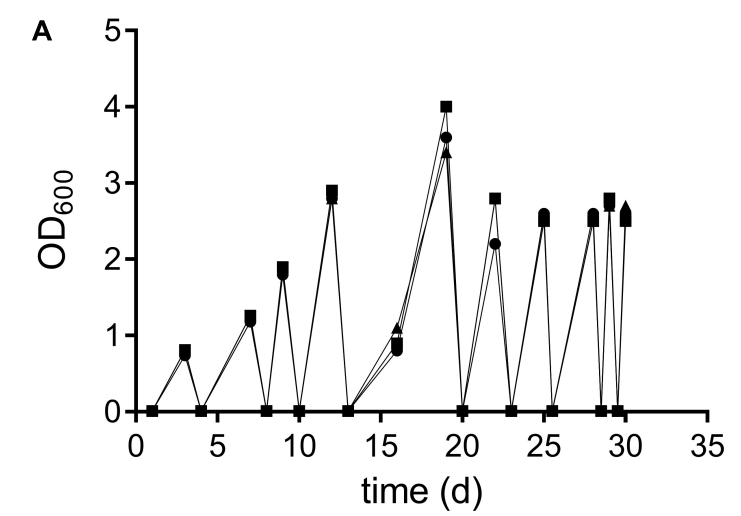

C

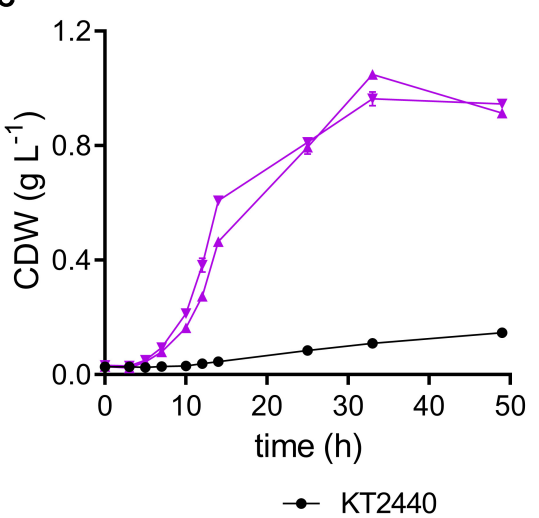

D

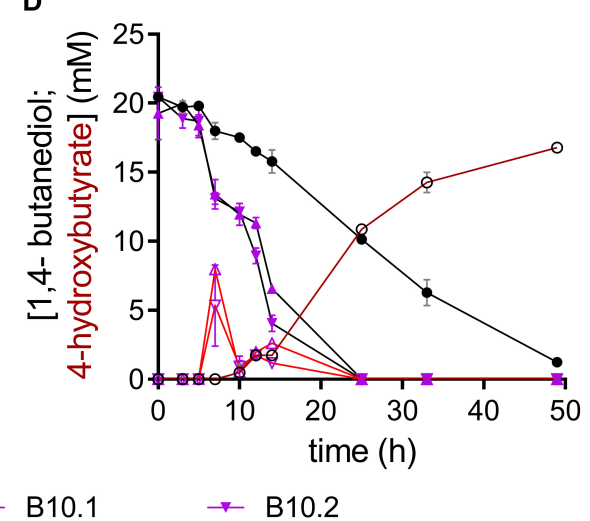

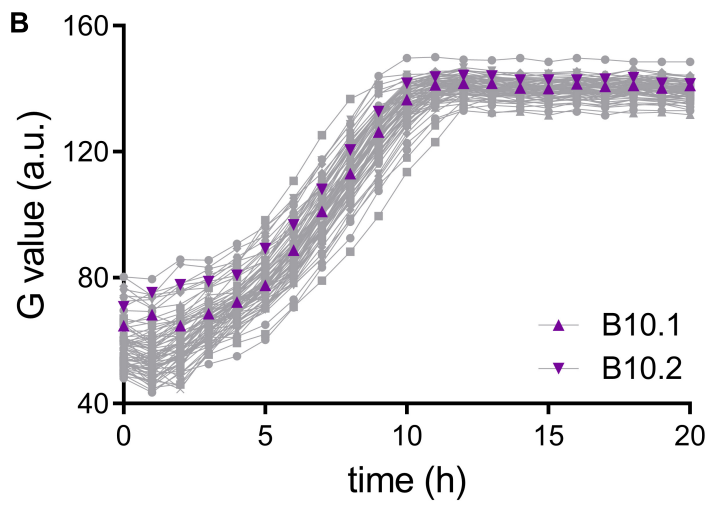

E

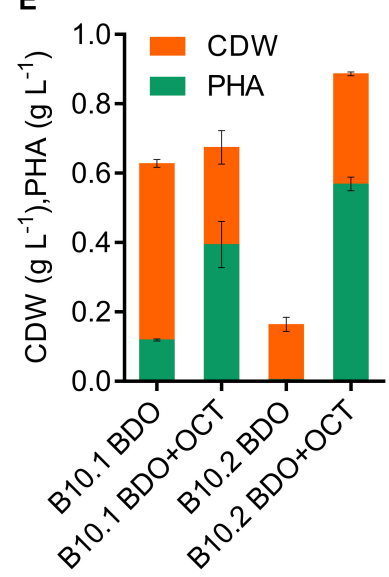

FIGURE 1 | Adaptive laboratory evolution of $P$. putida KT2440 on 1,4-butanediol. (A) Three parallel sequential batch cultivations on MSM with 20 mM 1,4-butanediol. (B) Growth of single strains isolated from each ALE batch on MSM with 20 mM 1,4-butanediol. The strains B10.1 (purple triangle) and B10.2 (purple inverted triangle) were selected for further investigation. Growth was detected via a Growth Profiler ${ }^{\circledR}$ using a 96-well plate. (C) Biomass growth and (D) 1,4-butanediol (closed symbols, black lines) and 4-hydroxybutyrate (open symbols, red lines) measured in cultures of the wildtype and evolved strains B10.1 and B10.2 in MSM with 20 mM 1,4-butanediol. (E) Growth and PHA formation of strains B10.1 and B10.2 cultivated for $48 \mathrm{~h}$ in nitrogen-limited MSM medium with 80 mM 1,4-butanediol (BDO), supplemented with or without 20 mM octanoic acid (OCT) after 24 h. Cultures took place in 250 mL Erlenmeyer shake flasks with a culture volume of $50 \mathrm{~mL}$. Error bars indicate the deviation of the mean $(n=2)$.

is likely caused by the high degree of reduction of butanediol, providing considerable reducing equivalents especially in the initial oxidation reactions ( $\mathrm{Li}$ et al., 2019). In contrast to the wildtype, the evolved strains only transiently accumulate low concentrations of 4-hydroxybutyrate, which are rapidly metabolized within $4 \mathrm{~h}$ (Figure 1). All 1,4-butanediol and derivatives that could be detected by HPLC were consumed within $25 \mathrm{~h}$, although biomass still increased significantly beyond this point. Other intermediates not detected by HPLC, possibly the lactone of 4-hydroxybutyrate, could likely accumulate transiently in the cultures of the evolved strains. However, the high biomass yield suggests that all carbon source was consumed at the end of the culture.

In order to assess the applicability of these evolved strains in a bio-upcycling approach, they were cultured in a nitrogenlimited MSM medium with $80 \mathrm{mM}$ 1,4-butanediol. Further cultures were supplemented with $20 \mathrm{mM}$ octanoic acid after $24 \mathrm{~h}$. These conditions enable the production of polyhydroxyalkanoate (PHA) from a (co-)feed of 1,4-butanediol. Without octanoic acid co-feed, strain B10.1 strains reached a final biomass concentration of $0.63 \pm 0.11 \mathrm{~g} \mathrm{~L}^{-1}$, of which $19 \%(0.12 \pm 0.003 \mathrm{~g}$
$\mathrm{L}^{-1}$ ) is PHA. Surprisingly, strain B10.2 reached a much lower biomass density, with only $3 \%$ PHA. With an octanoic acid cofeed, strain B10.2 reached the highest biomass concentrations of $0.89 \pm 0.005 \mathrm{~g} \mathrm{~L}^{-1}$, of which $64 \%\left(0.57 \pm 0.02 \mathrm{~g} \mathrm{~L}^{-1}\right)$ is PHA (Figure 1). This proves that 1,4-butanediol can be used as a (co-)substrate for the production of a value-added biopolymer, thereby in principle enabling the upcycling of e.g., hydrolyzed PU or polyester waste.

Evolution successfully yielded strains with a 4 - or 3.7fold improved growth rate on 1,4-butanediol compared to the wildtype. The fact that the wildtype accumulates much more 4hydroxybutyrate than the evolved strains indicates that this is likely the main metabolic bottleneck which was affected by ALE.

\section{Systems Analysis of 1,4-Butanediol Degradation in $P$. putida KT2440}

To investigate the molecular basis of their enhanced growth on 1,4-butanediol, the genomes of the evolved strains B10.1 and B10.2 were resequenced (NCBI SRA accession number SRP148839). The sequences were compared to our laboratory 
wildtype (SRX4119395) and a reference database genome of P. putida KT2440 (Belda et al., 2016, AE015451.2). A comparison of the latter two was previously described in the context of ethylene glycol metabolism in P. putida (Li et al., 2019). Therefore, we focus here only on differences between our laboratory wildtype and the B10 strains. In the evolved strains B10.1 and B10.2, seven and eight mutations, respectively, were identified in addition to the mutations already present in the laboratory wildtype. Most of these mutations were either silent or intergenic. In addition to these, in the genome of B10.1, an in-frame deletion of 69 bp was found in PP_2139, encoding DNA topoisomerase I. Since this enzyme is related to DNA replication and repair (Wang, 2002), this alteration is unlikely to affect 1,4-butanediol metabolism specifically. However, this mutation might still be favorable in a general sense by affecting growth rate through DNA replication. Furthermore, a missense mutation was identified B10.2 that affects PP_2889, encoding the transmembrane anti-sigma factor PrtR (Calero et al., 2018; Supplementary Table S3). An amino exchange (A240G, GCG/GGG) in this regulator, involved in temperature-related protease production (Burger et al., 2000), might enhance tolerance toward 1,4-butanediol and its oxidation products. Both of these mutations are likely related to general ALE effects selecting for faster growth or higher tolerance to chemical stressors, rather than affecting the operation of the metabolic network.

The PP_2046 gene, encoding for a LysR-type transcriptional regulator, stood out for being mutated in both evolved strains, with each carrying a different mutation. In B10.1, a nonsense mutation caused the loss of the start codon (ATG/ATA), while in B10.2 a missense mutation caused an amino acid exchange (E34G, GAG/GGG) in the helix-turn-helix DNA binding domain of the regulator (Supplementary Figure S1 and Supplementary Table S3). The mutations in this regulator likely affect the expression of the adjacent operon PP_2047-51 which encodes an iron-containing alcohol dehydrogenase, as well as enzymes involved in $\beta$-oxidation (Li et al., 2019).

In addition to the analysis of the changes on the genome level, proteomic analysis of the evolved strains and the wildtype during growth on glucose and 1,4-butanediol was conducted. This was done to identify relevant enzymes that are either constitutively expressed or natively induced by $1,4-$ butanediol. Three biological samples from each strain and culture condition, either grown on glucose or 1,4-butanediol in MSM, were harvested at mid-log phase (Supplementary Figure S2). The samples were normalized using total protein concentration to give the same starting protein concentration for all replicates.

In total, 2122 proteins were identified across all samples and growth conditions, representing $40 \%$ of the P. putida KT2440 proteome. The identified proteins exhibited a wide range of annotated biophysical (molecular mass, isoelectric point), biochemical (functional annotations) and structural (domains) properties, suggesting that the analysis was not biased in favor of, or against, any protein class.

When cultivated on 1,4-butanediol the evolved strain B10.1 expressed 19 proteins which were not present in the wildtype, while 313 proteins were up- or downregulated at least twofold compared to the wildtype. When evolved strain B10.2 was compared to the wildtype, 138 proteins showed at least two-fold difference in expression. The two evolved strains differed in their expression of 126 proteins. A large fraction of the differentially expressed proteins have no known function (Figure 2 and Supplementary Data File S2). The second-largest group can be categorized in amino acid metabolism and transport according to the clusters of orthologous groups (COG) classification (Tatusov et al., 1997). The likely reason for this large number of proteins with different expression levels is a large difference in growth rate of the B10 strains and the wildtype on 1,4-butanediol and the turnover of proteins during growth.

The top three highest expressed proteins during growth on glucose as well as 1,4-butanediol were PedE (ethanol dehydrogenase - PP_2674), PedI (aldehyde dehydrogenase PP_2680) and Tu-B (PP_0452), an elongation factor which is involved in the regulation of protein synthesis by mediating aminoacyl tRNA into a free site of ribosomes (Noel and Whitford, 2016). The latter Tu-B is a general growth-associated protein (Klumpp et al., 2009). The former two proteins are encoded within the ped cluster (PP_2663-80) (Li et al., 2019). To focus on 1,4-butanediol metabolism, specific proteins with activities in putative catabolic pathways (Figure 3) and associated transport steps were further investigated.

Genome sequencing uncovered mutations in PP_2046. The corresponding protein was not detected in the proteome analysis, indicating no or a low basal expression below the detection limit of the applied method, which is not uncommon for transcriptional regulators. Proteins encoded by the downstream $\beta$-oxidation-related operon were strongly upregulated in the wildtype grown on 1,4-butanediol vs. glucose, including a 3hydroxyacyl-CoA dehydrogenase (PP_2047, 22-fold), an acylCoA dehydrogenase (PP_2048, 16-fold), an iron-containing alcohol dehydrogenase (PP_2049, 52-fold), and an acetyl-CoA acetyltransferase (PP_2051, 25-fold). The hypothetical protein PP_2050 was not detected. On top of this strong induction by 1,4-butanediol in the wildtype, the genes in this operon were even further induced by 2.4- to 3 -fold in the evolved strains compared to the wildtype (Figure 3 and Supplementary Data File S2).

Theoretically, 1,4-butanediol can be metabolized through three possible pathways, all branching off at the point of 4-hydroxybutyrate. This 4-hydroxybutyrate was rapidly formed in cultivations of wildtype P. putida KT2440 on 1,4-butanediol, and also accumulates transiently with the B10 strains (Figure 1). This shows that oxidation of 1,4-butanediol to 4-hydroxybutyrate via alcohol and aldehyde oxidases already occurs at a high rate in the wildtype. The high expression levels of PedE, PedH and PedI suggest that these enzymes are major players in these oxidation steps. Although the encoding genes were not affected by the ALE, and they are only marginally upregulated when the strains were grown with 1,4-butanediol in comparison with growth on glucose, they are constitutively expressed on a very high level. This indicates a considerable metabolic investment of $P$. putida to be prepared for alcohol and aldehyde oxidation. This rapid oxidation is especially important for tolerance against the 


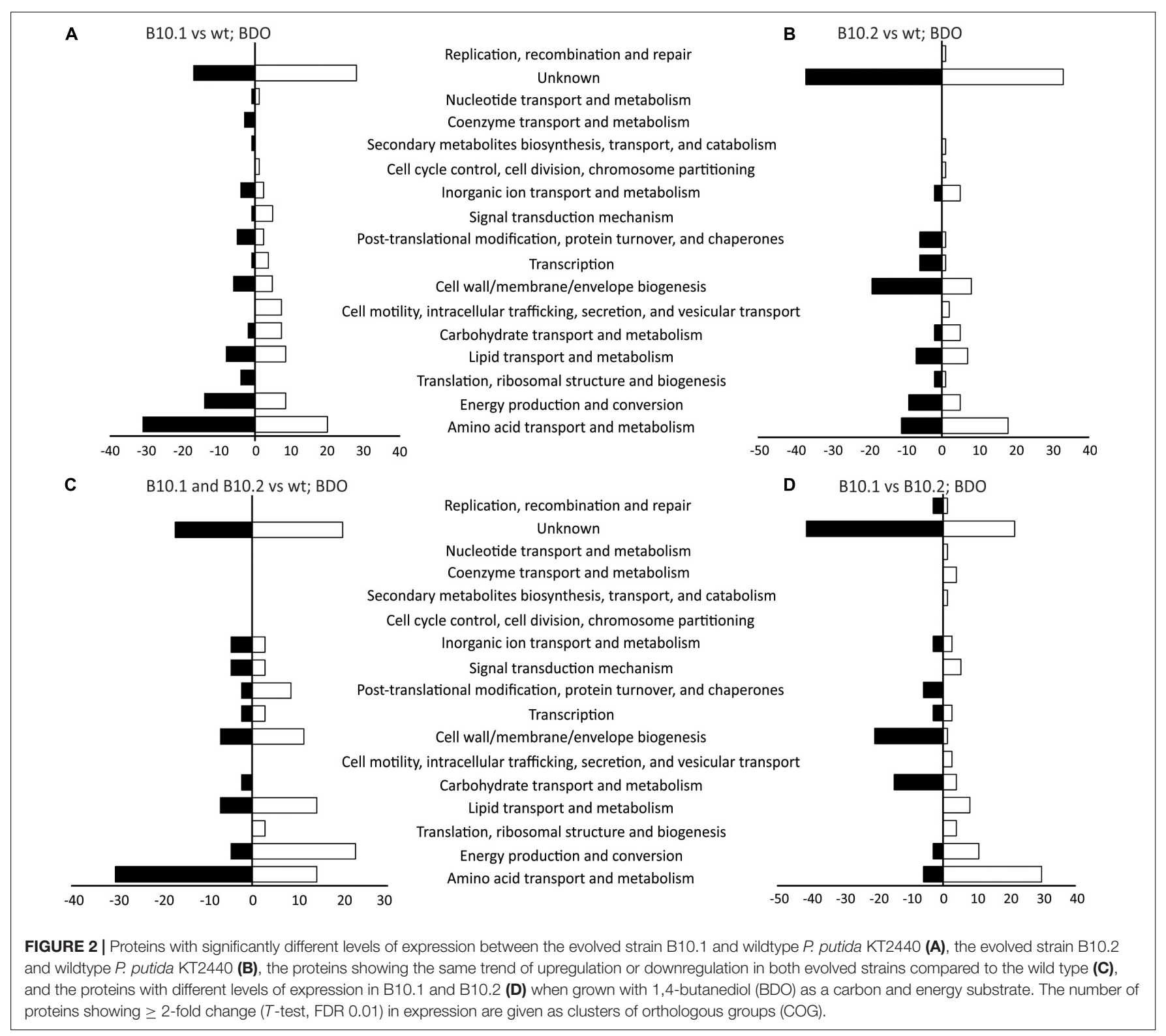

highly toxic aldehydes (Franden et al., 2018). Apparently, $P$. putida encounters such aldehydes often enough in its native environment to warrant this high constitutive expression. Besides these two enzymes, a number of other oxidoreductases are strongly induced upon growth on 1,4-butanediol vs. glucose. These include the GMC family oxidoreductase PP_0056 (BetA-I, along with its associated transporter PP_0057), the iron-containing alcohol dehydrogenase PP_2049, the 3-hydroxybutyrate dehydrogenase PP_3073 ( $\mathrm{HbdH})$, the isoquinoline oxidoreductase PP_3621-3 (IorAB-adhB), and the aldehyde dehydrogenase PP_5258 (amaB).

The resulting 4-hydroxybutyrate can theoretically be further oxidized by the same enzymes. However, the presence of a negatively charged carboxylate moiety likely affects substrate binding and it is more plausible that one or several other oxidoreductases with annotated activities on molecules with a carboxylate group similar to 4-hydroxybutyrate perform these oxidations. Several of such enzymes were upregulated during growth on 1,4-butanediol vs. glucose, including a 3hydroxyisobutyrate dehydrogenase (PP_4666, MmsB) and a methylmalonate semialdehyde dehydrogenase (PP_4667 MmsAII) (Steele et al., 1992; Zhou et al., 2013) for the alcohol oxidation step. The resulting succinate semialdehyde can be oxidized by the annotated succinate semialdehyde dehydrogenases PP_0213 (GabD-I) and PP_3151 (SadI), but also possibly by the methylmalonate-semialdehyde dehydrogenase PP_4667 (MmsAII). The resulting oxidation product succinate can be further metabolized in the TCA cycle. The low growth rate of the wildtype indicates that, although multiple putative proteins for both activities are expressed at a high level, these latter two oxidations only occur at a low rate at best, with a likely bottleneck in the oxidation of 4-hydroxybutyrate. 
A

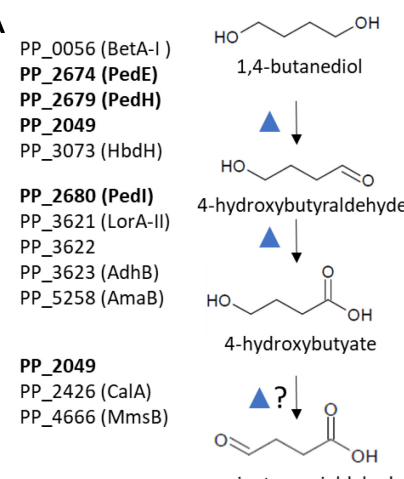

PP_0213 (GabD-I) PP_3151 (Sad-II)
PP_4667 (MmsA-II)

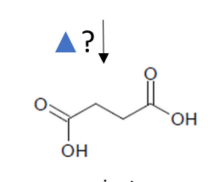

succinate

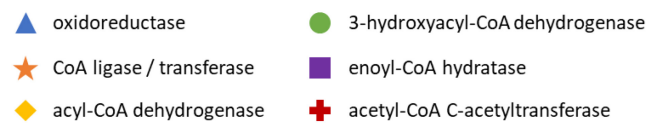

PP_2351 (PrpE)

PP_3122 (AtoA)

PP_3123 (AtoB)

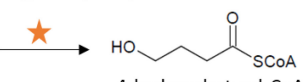

4-hydroxybutyryl-CoA

PP_1893 (FadE)

PP_2047
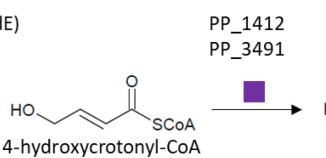

$\underbrace{\mathrm{SO}}_{\text {3,4-hydroxybutyryl-COA }}$
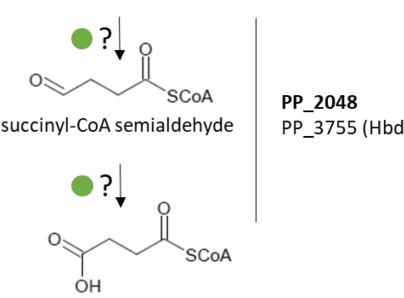

succinyl-CoA

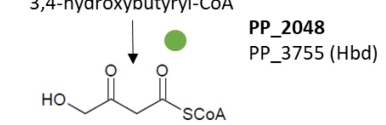

4-hydroxy-3-keto-butyryl-CoA

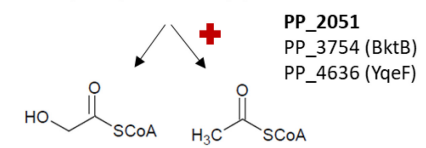

glycolyl-CoA acetyl-CoA
B

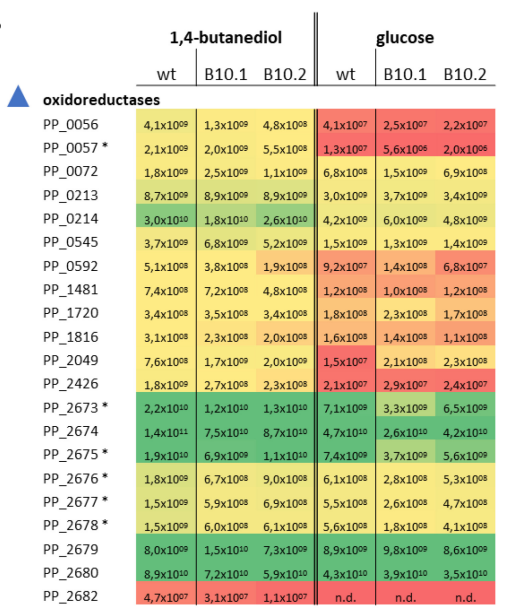

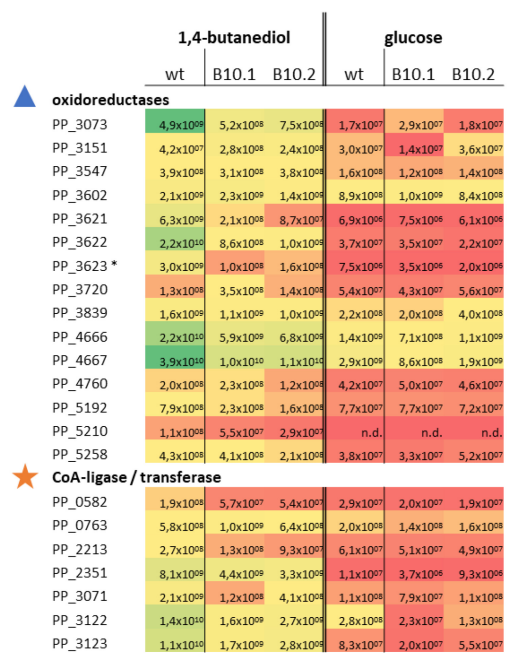

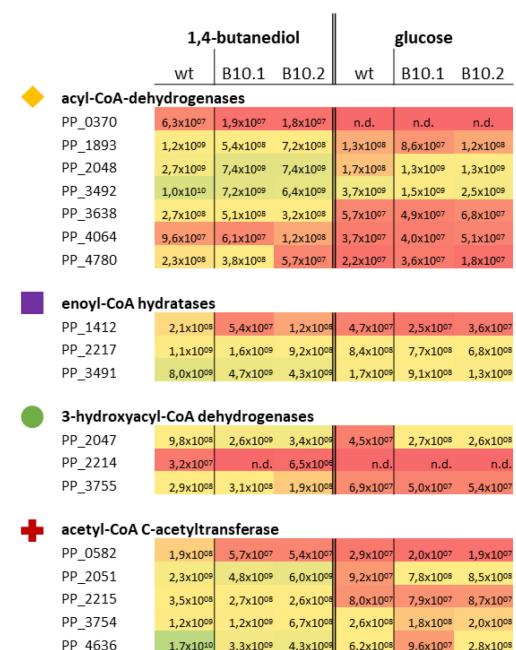

FIGURE 3 | Hypothetical pathways for 1,4-butanediol metabolism (A) and expression levels (Label Free Quantification, LFQ; Tyanova et al., 2016) representing the sum of the ion signal recorded in the mass spectrometer for all peptides derived from each protein of the corresponding proteins (B). Colors correspond to LFQ levels, red for low, yellow for average, and green for high values. Proteins which are strongly upregulated in response to growth on MSM with 1,4-butanediol compared to growth with glucose, or which have high expression level in all tested conditions, are indicated in (A), with proteins further investigated in this work in bold. Protein expression levels (B) of selected proteins for P. putida KT2440 and the evolved strains B10.1 and B10.2 growing in MSM with glucose or 1,4-butanediol are listed as oxidoreductases (blue triangle), CoA-ligases (orange star), acyl-CoA dehydrogenases (yellow diamond), 3-hydroxyacyl-CoA dehydrogenase (green circle), enoyl-CoA hydratases (purple rectangle), and acetyl-CoA C-actyltransferase (red cross). n/d, not detected. In case of operons, associated transporters or accessory proteins are included, marked with *.

As an alternative hypothesis to this direct oxidation route, 4-hydroxybutyrate can also be CoA-activated via CoA ligases or transferases. After CoA activation, 4-hydroxybutyryl-CoA can undergo $\beta$-oxidation, possibly through enzymes encoded by the PP_2047-51 operon downstream of PP_2046, which would result in glycolyl-CoA and acetyl-CoA (Figure 4). Other $\beta$-oxidation related proteins were also identified by the proteome analysis. Glycolyl-CoA may be converted into glycolate and subsequently metabolized through native pathways of P. putida (Li et al., 2019). Theoretically, 4-hydroxybutyrylCoA could also be further oxidized by acyl-CoA dehydrogenases to generate succinyl-CoA, provided that these have a side activity on the 4-hydroxy group instead of their usual 3-hydroxylated substrates.

Also of note was the relatively strong differential expression of genes related to the metabolism and transport of amines. The PP_0411-4 operon was highly expressed in the wildtype, but not in the evolved strains, during growth on 1,4-butanediol (Supplementary Data File S2). This operon encodes a polyamine $\mathrm{ABC}$ transporter for spermidine and putrescine, which are structurally and chemically similar to 1,4-butanediol. In spite of this large differential expression, no genomic mutations were 


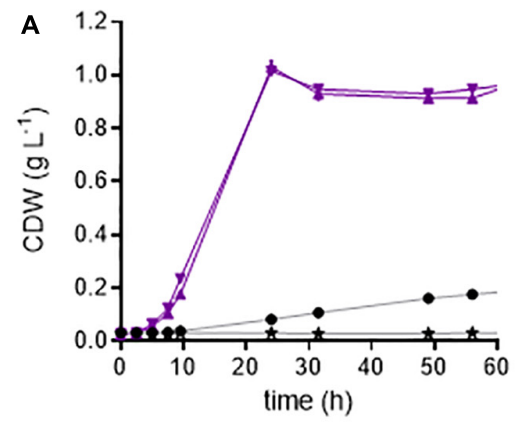

D

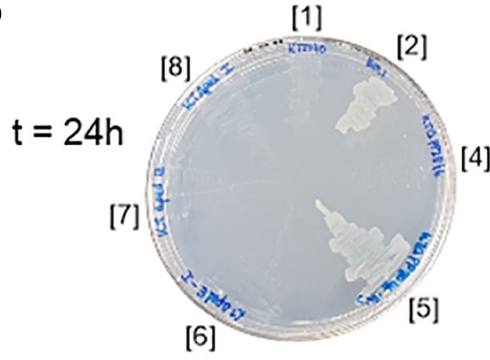

E

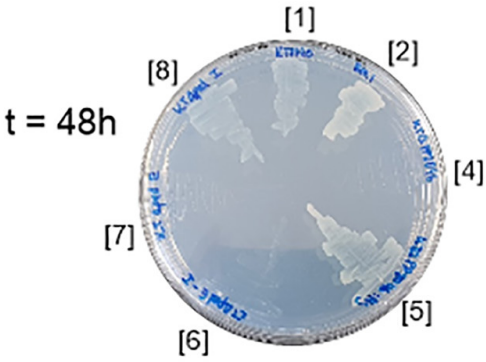

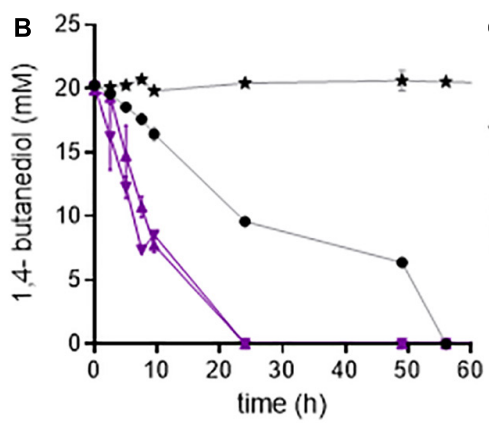

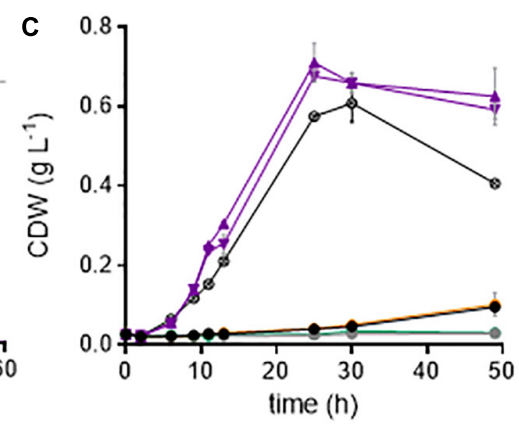

[1]

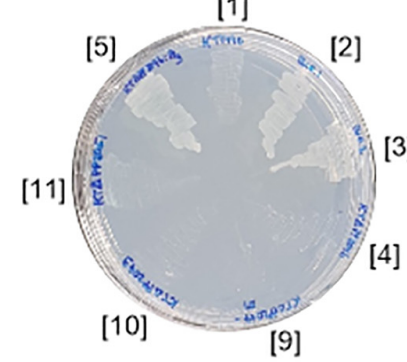

[3]

\begin{tabular}{|c|c|c|}
\hline no & symbol & strain \\
\hline 1 & $\rightarrow$ & KT2440 \\
\hline 2 & -1 & B10.1 \\
\hline 3 & $\rightarrow-$ & B10.2 \\
\hline 4 & - & KT2440 $\triangle P P \_2046$ \\
\hline 5 & - & KT2440 $\triangle P P$ 2046::14g \\
\hline 6 & $\star$ & KT2440 $\Delta p e d E-I$ \\
\hline 7 & & KT2440 $\Delta p e d E$ \\
\hline 8 & & KT2440 $\Delta p e d l$ \\
\hline 9 & & KT2440 $\triangle P P \_2047-51$ \\
\hline 10 & $\because$ & KT2440 $\Delta$ PP_2049 \\
\hline 11 & $\because$ & 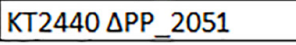 \\
\hline
\end{tabular}

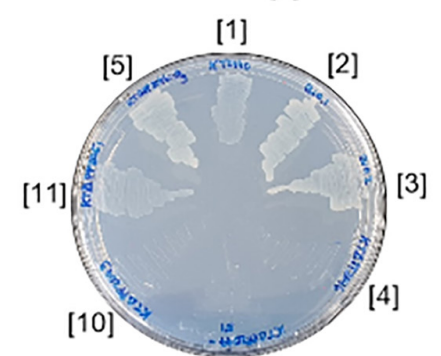

[9]

FIGURE 4 | Analysis of knockout strains of $P$. putida KT2440. Biomass growth (A) and 1,4-butanediol concentrations (B) of the wildtype, evolved strains and the $\Delta$ pedE-I knockout cultivated in shake flasks in MSM with 20 mM 1,4-butanediol. Growth of selected strains on 20 mM 4-hydroxybutyrate (C). Growth of selected strains on MSM agar plates with 20 mM 1,4-butanediol after 24 h (D) and 48 h (E). Strain numbers next to the plates correspond to full strain names listed in Table 1. The contrast of the images was increased by $20 \%$ to improve visibility. Error bars indicate the deviation of the mean $(n=2)$.

found in the evolved strains surrounding the operon, and the knockout of PP_0411-14 in P. putida KT2440 did not influence growth on 1,4-butanediol (data not shown). In contrast, operons encoding metabolic pathways for 4-aminobutanoate (PP_201315), ethanolamine (PP_0542-44), and ornithine (PP_09991001) were strongly upregulated on 1,4-butanediol vs. glucose (Supplementary Data File S2). The metabolism of some of these amines shares metabolic intermediates with the putative 1,4-butanediol pathways (Bandounas et al., 2011). Possibly, the high accumulation of 4-hydroxybutyrate in the wildtype induced the expression of these genes, leading to a misregulation during growth on 1,4-butanediol. Alternatively, one of the aldehyde intermediates may undergo amination, or the diamine transporter may facilitate uptake of 1,4-butanediol or its oxidation products.

\section{Pathway Validation}

The abovementioned genomic and proteomic analyses indicate several possible genes and enzymes that are either natively expressed at a high level, upregulated in the presence of 1,4butanediol, or activated by ALE. To test the relevance of these genes for 1,4-butanediol metabolism, several knockout strains were generated. The dehydrogenases encoded in the ped cluster (PP_2673-80) were constitutively expressed at a high level (Figure 3). To test the importance of these dehydrogenases to the degradation of 1,4-butanediol, the entire cluster $(\Delta p e d E$ $I)$, as well as individual genes pedE and pedI, were knocked out in $P$. putida KT2440. When $P$. putida KT2440 $\Delta$ pedE-I was cultivated in MSM with 1,4-butanediol no growth could be observed, nor was the substrate taken up or converted to 4hydroxybutyrate (Figure 4). Therefore, this cluster appears to be essential for the uptake and metabolism of 1,4-butanediol. The fact that no oxidation products were observed strongly suggests that these enzymes catalyze the initial oxidation steps.

The single knockouts of pedE and pedI were streaked on MSM plates containing $20 \mathrm{mM}$ 1,4-butanediol as sole carbon source. Of these knockouts, $P$. putida KT2440 $\Delta$ pedE did not grow, while the $\Delta$ pedI strain displayed growth similar to the wildtype after $48 \mathrm{~h}$ (Figure 4). Thus, the PQQ-dependent alcohol dehydrogenase 
A

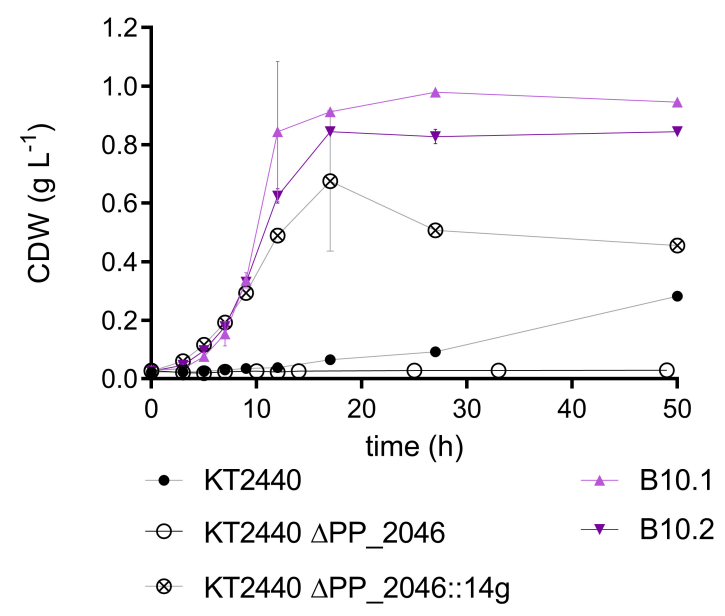

B

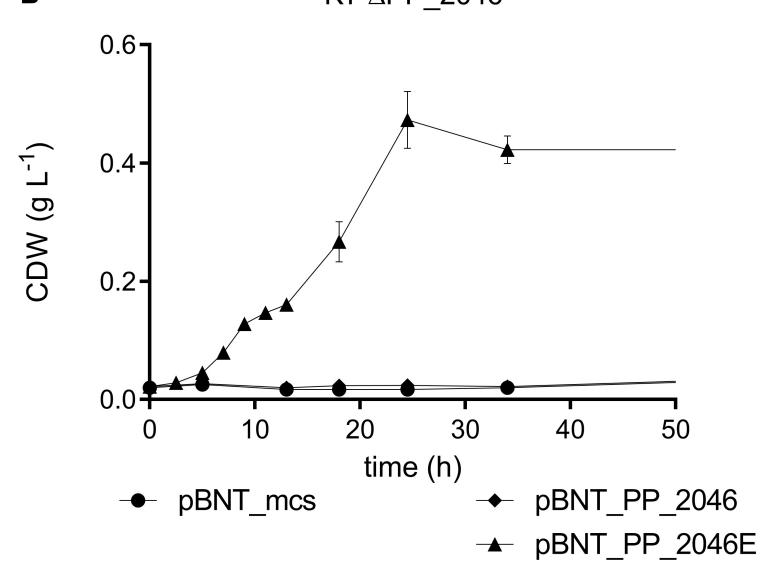

FIGURE 5 | (A) Biomass growth during the cultivation of P. putida KT2440 (black, circles), B10.1, B10.2 (purple, triangles), P. putida KT2440 $\Delta$ PP_2046 (black, circle) and P. putida KT2440 $\Delta$ PP_2046:14g (black, circled cross) in MSM medium with 20 mM 1,4-butanediol. (B) Biomass growth of P. putida KT2440 $\Delta$ PP_2046 transformants harboring an overexpressing construct for PP_2046 or PP_2046E and the empty vector cultivated in MSM with 20 mM 1,4-butanediol. Error bars indicate the deviation of the mean $(n=2)$.

PedE is likely responsible for the oxidation of 1,4-butanediol, while, surprisingly, the aldehyde dehydrogenase PedI does not seem to play an essential role in the further oxidation steps, likely because of the high redundancy of aldehyde dehydrogenases in P. putida. PedE, a homolog to ExaA from P. aeruginosa, is an extensively investigated pyrroloquinoline quinone alcohol dehydrogenase with a broad substrate activity, including 1butanol and 1,4-butanediol (Takeda et al., 2013). Furthermore, Wehrmann et al. (2017) showed activities of PedE toward structural similar alcohols and aldehydes of 1,4-butanediol, like 1-butanol and butyraldehyde. Additionally, the first steps of 1-butanol assimilation in P. putida BIRD-1 also involve homologs of the ped cluster (Simon et al., 2015; Vallon et al., 2015; Cuenca et al., 2016). The other dehydrogenases encoded within the ped cluster, PedH and also PedI, seem to be of minor relevance. PedE and $\mathrm{PedH}$ are both ethanol dehydrogenases but are inversely regulated by lanthanides. In the absent of those rare earth elements, pedE expression is induced and $p e d H$ is repressed (Wehrmann et al., 2017). Both PedE and PedH are highly expressed, but considering the absence of lanthanides, it is likely that PedH is not active.

The mutations found in PP_2046 and the upregulation of the adjacent operon PP_2047-51 strongly indicates an important role of the encoded enzymes. This operon contains an iron-containing alcohol dehydrogenase encoded by PP_2049 in addition to $\beta$-oxidation related genes. In literature, this dehydrogenase is placed in a context of $\beta$-oxidation, likely due to its association with the other genes in the PP_2047-51 operon (Poblete-Castro et al., 2012). However, PP_2049 is classified as an iron-containing alcohol dehydrogenase, which belongs to type III non-homologous $\mathrm{NAD}(\mathrm{P})^{+}$-dependent alcohol dehydrogenases (Mitchell et al., 2019). This family is known to have activity toward methanol, ethanol, propanol, and butanol (Hiu et al.,
1987; Gaona-López et al., 2016). It is highly likely that PP_2049 oxidizes one or more of the alcohol groups of 1,4-butanediol. Thus, neither direct oxidation to succinate nor $\beta$-oxidation can be ruled out by the observed mutation in PP_2046 and the upregulation of the associated operon. In order to determine the relevance of the operon and to distinguish between the effect of the alcohol dehydrogenase and the $\beta$-oxidation related genes, the operon and individual genes PP_2046, PP_2049, and PP_2051 were knocked out in wildtype P. putida KT2440 and in the evolved strains B10.1 and B10.2. Care was taken to avoid polar effects in the in-operon knockouts by leaving start and stop codons of overlapping genes intact using the pEMG system (Martínez-García and de Lorenzo, 2011). Deletion strains were tested for their ability to grow on MSM with 1,4-butanediol or 4-hydroxybutyrate as sole carbon source (Figure 4).

Both the wildtype and the evolved strains were unable to grow on 1,4-butanediol or 4-hydroxybutyrate when the regulator PP_2046, or the alcohol dehydrogenase PP_2049 were deleted (Figures 4, 5). The knockout of the whole operon also abolished growth. In contrast, deletion of PP_2051 did not affect growth on 1,4-butanediol (Supplementary Figure S3). This strongly suggests that PP_2049 is the main enzyme involved in the oxidation of 4-hydroxybutyrate. Both individual knockout strains of PP_2049 and pedE were unable to grow on 1,4-butanediol, making it unlikely that they oxidize the same substrate. More likely, the PP_2049 dehydrogenase is essential for the oxidation of 4-hydroxybutyrate, while PedE oxidizes 1,4-butanediol. Attempts to obtain direct biochemical evidence for the oxidation hypothesis with dehydrogenase assays on whole cell extracts of $P$. putida KT2440 wildtype, B10.1, and $\triangle P P \_2046$ with 4-hydroxybutyrate as a substrate were unsuccessful (Supplementary Figure S4) although this might be caused by instability of the PP_2049 enzyme and should 


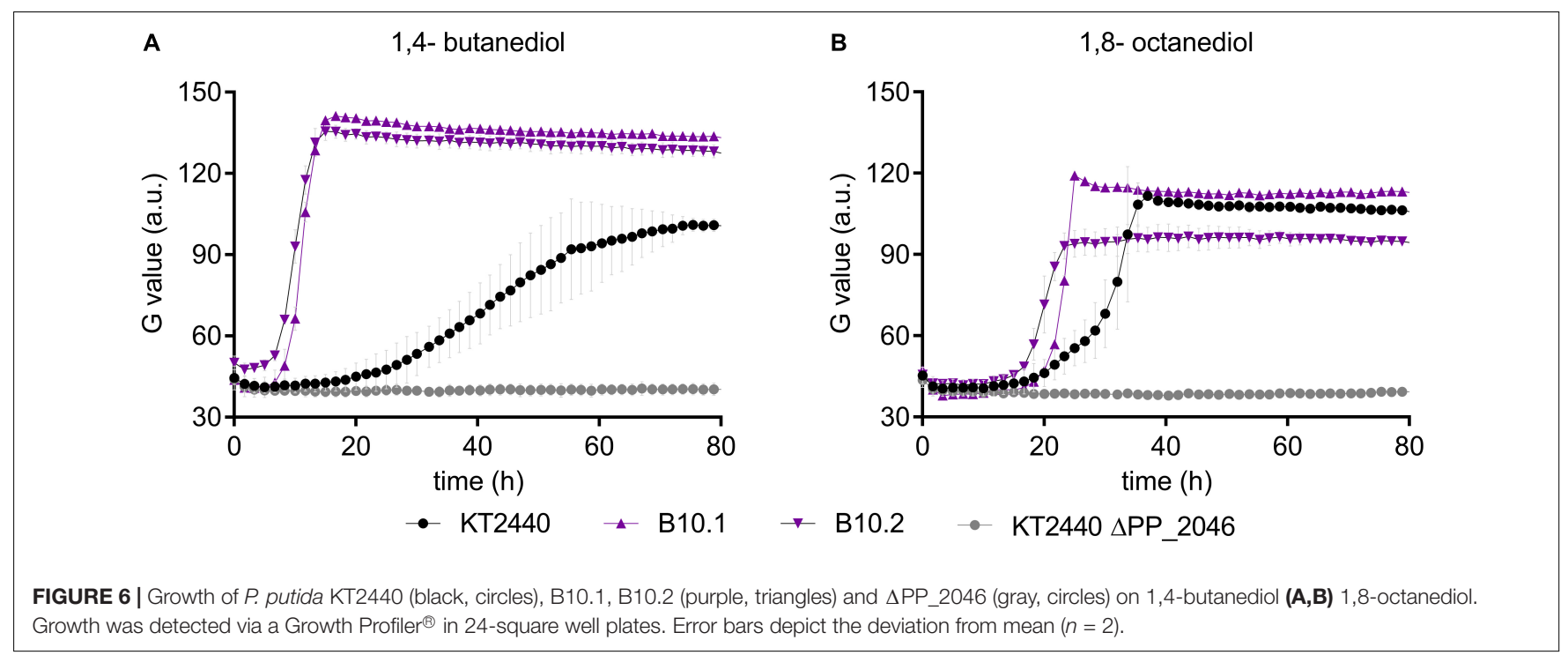

be investigated further. The lack of phenotype of the PP_2051 mutant suggests that $\beta$-oxidation is not involved, however, this is no clear proof since several other acetyl-CoA C-acetyltransferases are also expressed on a similar level (Figure 3).

The deletion of PP_2046 apparently causes a downregulation of the operon, while the mutations in PP_2046 in the evolved strains cause an overexpression of the adjacent operon. This is evident in the proteome data and also described in $\mathrm{Li}$ et al. (2019) for the E34G mutation in the context of ethylene glycol metabolism. In fact, the strains evolved on ethylene glycol also grow efficiently on 1,4-butanediol (Supplementary Figure S5). Expression in trans of the mutated regulator from B10.2 containing this mutation (denoted as PP_2046E) in P. putida KT2440 $\Delta$ PP_2046 enhanced growth of the wildtype on 1,4-butanediol, while expression of the native version could not restore growth (Figure 5). The regulator PP_2046 groups in the LysR-type family, which mainly contains transcriptional activators, repressors, and even dual function activators/repressors with a helix-turn-helix (HTH) DNAbinding domain at the N-terminus (Pérez-Rueda and ColladoVides, 2000; Maddocks and Oyston, 2008). Both mutations found in the B10.1 and B10.2 evolved strains are located in the first third of the gene, in the HTH domain. While the B10.1 version of PP_2046 lost its native start codon, alternatives start codons are present (Supplementary Figure S1). The fact that only the mutated version of PP_2046 can enable growth on 1,4butanediol, while its deletion abolishes growth, strongly suggests that this gene encodes an activator of the downstream operon, with an unknown inducer outside of the 1,4-butanediol context. It seems that a modification of the HTH domain is key to creating a constitutive activator.

In order to test whether overexpression of the PP_2047-51 operon alone was sufficient to enable faster growth on 1,4butanediol, PP_2046 was replaced by the strong constitutive promotor $\mathrm{P}_{14 g}$, facing the operon, resulting in the strain $P$. putida KT2440 $\Delta$ PP_2046:14g. Indeed, the growth on 1,4-butanediol was enhanced by 3.43 -fold compared to the wildtype (Figure 5).
This further indicates that the PP_2047-51 operon is the main determinant enabling fast growth on 1,4-butanediol. However, growth of this strain was somewhat slower than that of the evolved strains ( $\triangle$ PP_2046:14g: $0.249 \pm 0.004 \mathrm{~h}^{-1}$ ), indicating that other factors, possibly also regulated by PP_2046, are at play.

In this context, it should be noted that no gene encoding a CoA-ligase or transferase is present within the operon, which would be required for 1,4-butanediol degradation through $\beta$-oxidation. However, several of such enzymes were upregulated in the presence of 1,4-butanediol (Figure 3). To test whether the upregulation of this operon in the evolved strains enables enhanced $\beta$-oxidation, growth of the wildtype, the evolved strains, and $\triangle P P \_2046$ were analyzed on longer-chain $\alpha, \omega$-diols. The $\Delta$ PP_2046 strain did not grow on any of the tested diols. In contrast, both the evolved strain and the wildtype grew on 1,4butanediol and 1,8-octanediol, with the evolved strains growing at a significantly higher rate (Figure 6). Surprisingly, none of the strains grew on 1,6-hexanediol or 1,7-heptanediol. A similar trend of faster growth by the evolved strains was also observed on butanol (Supplementary Figure S6). Since these substrates can only be metabolized through $\beta$-oxidation, these results strongly suggest that the upregulation of the PP_2047-51 operon enables higher activity of this pathway, and they prove that PP_2046 is an essential regulator for the metabolism of these short- to medium-chain alcohols.

\section{CONCLUSION}

Adaptive laboratory evolution was successfully used to enhance growth of P. putida KT2440 on 1,4-butanediol. Putative degradation pathways of this important plastic monomer were contextualized with leads from genome resequencing and proteome analysis, which were verified by knockout and overexpression analyses and physiological data. The alcohol dehydrogenases PedE and PP_2049 were found to be essential for growth on 1,4-butanediol, with the latter also being required for 
growth on 4-hydroxybutyrate. Mutations in the transcriptional regulator PP_2046 were the main cause of enhanced growth in the ALE strains. The evolved phenotype could be reproduced through reverse engineering, either by overexpression of the PP_2047-51 operon by promoter exchange, or through in trans expression of the mutated regulator. In all, the knockout analysis favors the hypothesis of direct oxidation of 1,4-butanediol, via 4hydroxybutyrate, to succinate. However, the alternative $\beta$-oxidation hypothesis can't be ruled out, and possibly both pathways operate simultaneously.

\section{DATA AVAILABILITY STATEMENT}

The sequences have been deposited in the NCBI Sequence Read Archive (SRA) with the accession number SRP148839 for ethylene glycol ALE strains (including our laboratory wildtype SRX4119395 used in this study) and SRP148839 for the 1,4butanediol ALE strains. Raw data for all figures shown is available from the author upon reasonable request.

\section{AUTHOR CONTRIBUTIONS}

NW and LB conceived the study with the help of KO'C and TN. NW supervised the study with support of LB and KO'C. NW and W-JL designed the experiments. W-JL performed the experiments with the help of P-JN. TN performed the proteome analysis. W-JL and NW prepared the figures and wrote the manuscript with the help of all authors. All authors have read and approved the final version of this

\section{REFERENCES}

Bagdasarian, M., Lurz, R., Rückert, B., Franklin, F. C. H., Bagdasarian, M. M., Frey, J., et al. (1981). Specific-purpose plasmid cloning vectors II. Broad host range, high copy number, RSF 1010-derived vectors and a host-vector system for gene cloning in Pseudomonas. Gene 16, 237-247. doi: 10.1016/0378-1119(81) 90080-9

Bandounas, L., Ballerstedt, H., de Winde, J. H., and Ruijssenaars, H. J. (2011). Redundancy in putrescine catabolism in solvent tolerant Pseudomonas putida S12. J. Biotechnol. 154, 1-10. doi: 10.1016/j.jbiotec.2011.04.005

Basler, G., Thompson, M., Tullman-Ercek, D., and Keasling, J. (2018). A Pseudomonas putida efflux pump acts on short-chain alcohols. Biotechnol. Biofuels 11:136. doi: 10.1186/s13068-018-1133-9

Bednarz, A., Spieß, A. C., and Pfennig, A. (2017). Reactive and physical extraction of bio-based diamines from fermentation media. J. Chem. Technol. Biotechnol. 92, 1817-1824. doi: 10.1002/jctb.5183

Behrendt, G., and Naber, B. (2009). The chemical recycling of polyurethanes. J. Univ. Chem. Technol. Metall. 44, 3-23.

Belda, E., van Heck, R. G. A., José Lopez-Sanchez, M., Cruveiller, S., Barbe, V., Fraser, C., et al. (2016). The revisited genome of Pseudomonas putida KT2440 enlightens its value as a robust metabolic chassis. Environ. Microbiol. 18, 3403-3424. doi: 10.1111/1462-2920.13230

Brandl, H., Gross, R. A., Lenz, R. W., and Fuller, R. C. (1988). Pseudomonas oleovorans as a source of poly(beta-hydroxyalkanoates) for potential applications as biodegradable polyesters. Appl. Environ. Microbiol. 54, 1977-1982. doi: 10.1128/aem.54.8.1977-1982.1988

Burgard, A., Burk, M. J., Osterhout, R., van Dien, S., and Yim, H. (2016). Development of a commercial scale process for production of 1,4-butanediol manuscript, analyzed, and interpreted the data. SK oversaw PHA production experiments.

\section{FUNDING}

The RWTH and UCD researchers acknowledge funding from the European Union's Horizon 2020 Research and Innovation Programme under Grant Agreement No. 633962 for the project P4SB and No. 863922 for the project MIX-UP. NW was supported by the German Research Foundation through the Emmy Noether project WI 4255/1-1. The laboratory of LB was partially funded by the Deutsche Forschungsgemeinschaft (DFG, German Research Foundation) under Germany's excellence strategy within the clusters of excellence 236 "TMFB - TailorMade Fuels from Biomass" and 2186 "FSC - The Fuel Science Center." TN and KO'C acknowledge the funding form Science Foundation Ireland Research Centre Grant No. SFI/16/RC/3889.

\section{ACKNOWLEDGMENTS}

We gratefully acknowledge Janosch Klebensberger (University of Stuttgart), Gregg Beckham (NREL), Jörg Pietruszka (HHU Düsseldorf), as well as all P4SB partners, for helpful discussion.

\section{SUPPLEMENTARY MATERIAL}

The Supplementary Material for this article can be found online at: https://www.frontiersin.org/articles/10.3389/fmicb. 2020.00382/full\#supplementary-material

from sugar. Curr. Opin. Biotechnol. 42, 118-125. doi: 10.1016/j.copbio.2016. 04.016

Burger, M., Woods, R. G., McCarthy, C., and Beacham, I. R. (2000). Temperature regulation of protease in Pseudomonas fluorescens LS107d2 by an ECF sigma factor and a transmembrane activator. Microbiology 146(Pt. 12), 3149-3155. doi: 10.1099/00221287-146-12-3149

Calero, P., Jensen, S. I., Bojanoviè, K., Lennen, R. M., Koza, A., and Nielsen, A. T. (2018). Genome-wide identification of tolerance mechanisms toward p-coumaric acid in Pseudomonas putida. Biotechnol. Bioeng. 115, 762-774. doi: 10.1002/bit.26495

Chomczynski, P., and Rymaszewski, M. (2006). Alkaline polyethylene glycol-based method for direct PCR from bacteria, eukaryotic tissue samples, and whole blood. Biotechniques 40, 454, 456, 458. doi: 10.2144/000112149

Cox, J., and Mann, M. (2008). MaxQuant enables high peptide identification rates, individualized p.p.b.-range mass accuracies and proteome-wide protein quantification. Nat. Biotechnol. 26, 1367-1372. doi: 10.1038/nbt.1511

Cox, J., Neuhauser, N., Michalski, A., Scheltema, R. A., Olsen, J. V., and Mann, M. (2011). Andromeda: a peptide search engine integrated into the MaxQuant environment. J. Proteome Res. 10, 1794-1805. doi: 10.1021/Pr101065j

Cuenca, M. D. S., Roca, A., Molina-Santiago, C., Duque, E., Armengaud, J., GómezGarcia, M. R., et al. (2016). Understanding butanol tolerance and assimilation in Pseudomonas putida BIRD-1: an integrated omics approach. Microbiol. Biotechnol. 9, 100-115. doi: 10.1111/1751-7915.12328

Danso, D., Chow, J., and Streit, W. R. (2019). Plastics: environmental and Biotechnological Perspectives on Microbial Degradation. Appl. Environ. Microbiol. 85:e01095-19. doi: 10.1128/AEM.01095-19

DePristo, M. A., Banks, E., Poplin, R., Garimella, K. V., Maguire, J. R., Hartl, C., et al. (2011). A framework for variation discovery and genotyping using 
next-generation DNA sequencing data. Nat. Genet. 43, 491-498. doi: 10.1038/ ng.806

Dragosits, M., and Mattanovich, D. (2013). Adaptive laboratory evolution principles and applications for biotechnology. Microb. Cell Fact. 12:64. doi: 10.1186/1475-2859-12-64

Franden, M. A., Jayakody, L. N., Li, W.-J., Wagner, N. J., Cleveland, N. S., Michener, W. E., et al. (2018). Engineering Pseudomonas putida KT2440 for efficient ethylene glycol utilization. Metab. Eng. 48, 197-207. doi: 10.1016/j.ymben.2018. 06.003

Gaona-López, C., Julián-Sánchez, A., and Riveros-Rosas, H. (2016). Diversity and Evolutionary Analysis of Iron-Containing (Type-III) Alcohol Dehydrogenases in Eukaryotes. PLoS One 11:e0166851. doi: 10.1371/journal.pone.0166851

Gibson, D. G., Young, L., Chuang, R.-Y., Venter, J. C., Hutchison, C. A., and Smith, H. O. (2009). Enzymatic assembly of DNA molecules up to several hundred kilobases. Nat. Methods 6, 343-345. doi: 10.1038/nmeth.1318

Grand View Research (2017). 1,4-Butanediol (BDO) Market Report 1,4-Butanediol (BDO) Market Analysis By Application (Tetrahydrofuran (THF), Polybutylene Terephthalate (PBT), Gamma-Butyrolactone (GBL), Polyurethane (PU)), By Region (North America, Europe, Asia Pacific, CSA, MEA), And Segment Forecasts, 2018 - 2025. San Francisco, CA: Grand View Research, Inc.

Guzik, M. W., Kenny, S. T., Duane, G. F., Casey, E., Woods, T., Babu, R. P., et al. (2014). Conversion of post consumer polyethylene to the biodegradable polymer polyhydroxyalkanoate. Appl. Microbiol. Biotechnol. 98, 4223-4232. doi: 10.1007/s00253-013-5489-2

Hanahan, D. (1983). Studies on transformation of Escherichia coli with plasmids. J. Mol. Biol. 166, 557-580. doi: 10.1016/S0022-2836(83)80284-8

Hartmans, S., Smiths, J. P., van der Werf, M. J., Volkering, F., and de Bont, J. A. M. (1989). Metabolism of styrene oxide and 2-phenylethanol in the styrenedegrading Xanthobacter strain 124X. Appl. Environ. Microbiol. 55, 2850-2855. doi: 10.1128/aem.55.11.2850-2855.1989

Hiu, S. F., Zhu, C. X., Yan, R. T., and Chen, J. S. (1987). Butanol-ethanol dehydrogenase and butanol-ethanol-isopropanol dehydrogenase: different alcohol dehydrogenases in two strains of Clostridium beijerinckii (Clostridium butylicum). Appl. Environ. Microbiol. 53, 697-703. doi: 10.1128/aem.53.4.697703.1987

Howard, G. T. (2002). Biodegradation of polyurethane: a review. Int. Biodeterior. Biodegradation 49, 245-252. doi: 10.1016/S0964-8305(02)00 051-3

Huang, D. W., Sherman, B. T., and Lempicki, R. A. (2009a). Bioinformatics enrichment tools: paths toward the comprehensive functional analysis of large gene lists. Nucleic Acids Res. 37, 1-13. doi: 10.1093/nar/gkn923

Huang, D. W., Sherman, B. T., and Lempicki, R. A. (2009b). Systematic and integrative analysis of large gene lists using DAVID bioinformatics resources. Nat. Protoc. 4, 44-57. doi: 10.1038/nprot.2008.211

Huerta-Cepas, J., Szklarczyk, D., Forslund, K., Cook, H., Heller, D., Walter, M. C., et al. (2016). eggNOG 4.5: a hierarchical orthology framework with improved functional annotations for eukaryotic, prokaryotic and viral sequences. Nucleic Acids Res. 44, D286-D293. doi: 10.1093/nar/gkv1248

Hung, C.-S., Zingarelli, S., Nadeau, L. J., Biffinger, J. C., Drake, C. A., Crouch, A. L., et al. (2016). Carbon catabolite repression and impranil polyurethane degradation in Pseudomonas protegens Strain Pf-5. Appl. Environ. Microbiol. 82, 6080-6090. doi: 10.1128/AEM.01448-16

Kagi, J. H., and Vallee, B. H. (1960). The role of zinc in alcohol dehydrogenase. $\mathrm{V}$. The effect of metal-binding agents on the structure of the yeast alcohol dehydrogenase molecule. J. Biol. Chem. 235, 3188-3192.

Kenny, S. T., Runic, J. N., Kaminsky, W., Woods, T., Babu, R. P., Keely, C. M., et al. (2008). Up-Cycling of PET (Polyethylene Terephthalate) to the biodegradable plastic PHA (Polyhydroxyalkanoate). Environ. Sci. Technol. 42, 7696-7701. doi: 10.1021/es801010e

Kenny, S. T., Runic, J. N., Kaminsky, W., Woods, T., Babu, R. P., and O'Connor, K. E. (2012). Development of a bioprocess to convert PET derived terephthalic acid and biodiesel derived glycerol to medium chain length polyhydroxyalkanoate. Appl. Microbiol. Biotechnol. 95, 623-633. doi: 10.1007/ s00253-012-4058-4

Klumpp, S., Zhang, Z., and Hwa, T. (2009). Growth rate-dependent global effects on gene expression in bacteria. Cell 139, 1366-1375. doi: 10.1016/j.cell.2009. 12.001
Lageveen, R. G., Huisman, G. W., Preusting, H., Ketelaar, P., Eggink, G., and Witholt, B. (1988). Formation of polyesters by Pseudomonas oleovorans: effect of substrates on formation and composition of poly-(R)-3-hydroxyalkanoates and poly-(R)-3-hydroxyalkenoates. Appl. Environ. Microbiol. 54, 2924-2932. doi: 10.1128/aem.54.12.2924-2932.1988

Lennen, R. M., Jensen, K., Mohammed, E. T., Malla, S., Börner, R. A., Chekina, K., et al. (2019). Adaptive laboratory evolution reveals general and specific chemical tolerance mechanisms and enhances biochemical production. bioRxiv [Preprint]. doi: 10.1101/634105

Li, H., and Durbin, R. (2009). Fast and accurate short read alignment with BurrowsWheeler transform. Bioinformatics 25, 1754-1760. doi: 10.1093/bioinformatics/ btp324

Li, W.-J., Jayakody, L. N., Franden, M. A., Wehrmann, M., Daun, T., Hauer, B., et al. (2019). Laboratory evolution reveals the metabolic and regulatory basis of ethylene glycol metabolism by Pseudomonas putida KT2440. Environ. Microbiol. 21, 3669-3682. doi: 10.1111/1462-2920.14703

Liu, H., and Lu, T. (2015). Autonomous production of 1,4-butanediol via a de novo biosynthesis pathway in engineered Escherichia coli. Metab. Eng. 29, 135-141. doi: 10.1016/j.ymben.2015.03.009

Maddocks, S. E., and Oyston, P. C. F. (2008). Structure and function of the LysR-type transcriptional regulator (LTTR) family proteins. Microbiology 154, 3609-3623. doi: 10.1099/mic.0.2008/022772-0

Magnin, A., Hoornaert, L., Pollet, E., Laurichesse, S., Phalip, V., and Avérous, L. (2019a). Isolation and characterization of different promising fungi for biological waste management of polyurethanes. Microbiol. Biotechnol. 12, 544555. doi: 10.1111/1751-7915.13346

Magnin, A., Pollet, E., Perrin, R., Ullmann, C., Persillon, C., Phalip, V., et al. (2019b). Enzymatic recycling of thermoplastic polyurethanes: synergistic effect of an esterase and an amidase and recovery of building blocks. Waste Manag. 85, 141-150. doi: 10.1016/j.wasman.2018.12.024

Martínez-García, E., Aparicio, T., Lorenzo, V., de, and Nikel, P. I. (2014). New transposon tools tailored for metabolic engineering of gram-negative microbial cell factories. Front. Bioeng. Biotechnol. 2:46. doi: 10.3389/fbioe.2014.00046

Martínez-García, E., and de Lorenzo, V. (2011). Engineering multiple genomic deletions in Gram-negative bacteria: analysis of the multi-resistant antibiotic profile of Pseudomonas putida KT2440. Environ. Microbiol. 13, 2702-2716. doi: 10.1111/j.1462-2920.2011.02538.x

McKenna, A., Hanna, M., Banks, E., Sivachenko, A., Cibulskis, K., Kernytsky, A., et al. (2010). The genome analysis toolkit: a MapReduce framework for analyzing next-generation DNA sequencing data. Genome Res. 20, 1297-1303. doi: $10.1101 /$ gr.107524.110

Mitchell, A. L., Attwood, T. K., Babbitt, P. C., Blum, M., Bork, P., Bridge, A., et al. (2019). InterPro in 2019: improving coverage, classification and access to protein sequence annotations. Nucleic Acids Res. 47, D351-D360. doi: 10.1093/ nar/gky1100

Narancic, T., and O'Connor, K. E. (2017). Microbial biotechnology addressing the plastic waste disaster. Microbiol. Biotechnol. 10, 1232-1235. doi: 10.1111/17517915.12775

Narancic, T., Scollica, E., Kenny, S. T., Gibbons, H., Carr, E., Brennan, L., et al. (2016). Understanding the physiological roles of polyhydroxybutyrate (PHB) in Rhodospirillum rubrum $\mathrm{S} 1$ under aerobic chemoheterotrophic conditions. Appl. Microbiol. Biotechnol. 100, 8901-8912. doi: 10.1007/s00253-016-7711-5

Nelson, K. E., Weinel, C., Paulsen, I. T., Dodson, R. J., Hilbert, H., Martins, et al. (2002). Complete genome sequence and comparative analysis of the metabolically versatile Pseudomonas putida KT2440. Environ. Microbiol. 4, 799-808.

Nikel, P. I., and de Lorenzo, V. (2018). Pseudomonas putida as a functional chassis for industrial biocatalysis: from native biochemistry to trans-metabolism. Metab. Eng. 50, 142-155. doi: 10.1016/j.ymben.2018.05.005

Nikel, P. I., Martínez-García, E., and de Lorenzo, V. (2014). Biotechnological domestication of Pseudomonads using synthetic biology. Nat. Rev. Microbiol. 12, 368-379. doi: 10.1038/nrmicro3253

Noel, J. K., and Whitford, P. C. (2016). How EF-Tu can contribute to efficient proofreading of aa-tRNA by the ribosome. Nat. Commun. 7:13314. doi: 10.1038/ ncomms13314

Parke, D., Garcia, M. A., and Ornston, L. N. (2001). Cloning and Genetic Characterization of dca Genes Required for -Oxidation of Straight-Chain 
Dicarboxylic Acids in Acinetobacter sp. Strain ADP1. Appl. Environ. Microbiol. 67, 4817-4827. doi: 10.1128/AEM.67.10.4817-4827.2001

Pérez-Rueda, E., and Collado-Vides, J. (2000). The repertoire of DNA-binding transcriptional regulators in Escherichia coli K-12. Nucleic Acids Res. 28, 18381847. doi: 10.1093/nar/28.8.1838

Poblete-Castro, I., Escapa, I. F., Jäger, C., Puchalka, J., Lam, C. M. C., Schomburg, D., et al. (2012). The metabolic response of P. putida KT2442 producing high levels of polyhydroxyalkanoate under single- and multiple-nutrient-limited growth: highlights from a multi-level omics approach. Microb. Cell Fact. 11:34. doi: 10.1186/1475-2859-11-34

Ramos, J. L., Duque, E., Gallegos, M.-T., Godoy, P., Ramos-Gonzalez, M. I., Rojas, A., et al. (2002). Mechanisms of solvent tolerance in gram-negative bacteria. Annu. Rev. Microbiol. 56, 743-768. doi: 10.1146/annurev.micro.56. 012302.161038

Rühl, J., Schmid, A., and Blank, L. M. (2009). Selected Pseudomonas putida strains able to grow in the presence of high butanol concentrations. Appl. Environ. Microbiol. 75, 4653-4656. doi: 10.1128/AEM.00225-09

Samanta, S. K., Singh, O. V., and Jain, R. K. (2002). Polycyclic aromatic hydrocarbons: environmental pollution and bioremediation. Trends Biotechnol. 20, 243-248. doi: 10.1016/S0167-7799(02)01943-1

Schmidt, J., Wei, R., Oeser, T., Dedavid, E., Silva, L. A., Breite, D., et al. (2017). Degradation of Polyester Polyurethane by Bacterial Polyester Hydrolases. Polymers 9:65. doi: 10.3390/polym9020065

Simon, O., Klebensberger, J., Mükschel, B., Klaiber, I., Graf, N., Altenbuchner, J., et al. (2015). Analysis of the molecular response of Pseudomonas putida KT2440 to the next-generation biofuel n-butanol. J. Proteom. 122, 11-25. doi: 10.1016/j.jprot.2015.03.022

Spini, G., Spina, F., Poli, A., Blieux, A.-L., Regnier, T., Gramellini, C., et al. (2018). ). Molecular and Microbiological Insights on the Enrichment Procedures for the Isolation of Petroleum Degrading Bacteria and Fungi. Front. Microbiol. 9:2543. doi: $10.3389 /$ fmicb. 2018.02543

Steele, M. I., Lorenz, D., Hatter, K., Park, A., and Sokatch, J. R. (1992). Characterization of the $m m s A B$ operon of Pseudomonas aeruginosa PAO encoding methylmalonate-semialdehyde dehydrogenase and 3-hydroxyisobutyrate dehydrogenase. J. Biol. Chem. 267, 13585-13592.

Tahseen, R., Arslan, M., Iqbal, S., Khalid, Z. M., and Afzal, M. (2019). Enhanced degradation of hydrocarbons by gamma ray induced mutant strain of Pseudomonas putida. Biotechnol. Lett. 41, 391-399. doi: 10.1007/s10529-01902644-y

Takeda, K., Matsumura, H., Ishida, T., Samejima, M., Igarashi, K., Nakamura, N., et al. (2013). The two-step electrochemical oxidation of alcohols using a novel recombinant PQQ alcohol dehydrogenase as a catalyst for a bioanode. Bioelectrochemistry 94, 75-78. doi: 10.1016/j.bioelechem.2013.08.001

Tatusov, R. L., Koonin, E. V., and Lipman, D. J. (1997). A genomic perspective on protein families. Science 278, 631-637. doi: 10.1126/science.278.5338.631

The UniProt Consortium (2019). UniProt: a worldwide hub of protein knowledge. Nucleic Acids Res. 47, D506-D515. doi: 10.1093/nar/gky1049

Thorvaldsdóttir, H., Robinson, J. T., and Mesirov, J. P. (2013). Integrative Genomics Viewer (IGV): high-performance genomics data visualization and exploration. Brief. Bioinform. 14, 178-192. doi: 10.1093/bib/bbs017

Tyanova, S., Temu, T., Sinitcyn, P., Carlson, A., Hein, M. Y., Geiger, T., et al. (2016). The perseus computational platform for comprehensive analysis of (prote)omics data. Nat. Methods 13, 731-740. doi: 10.1038/nmeth.3901

Vallon, T., Simon, O., Rendgen-Heugle, B., Frana, S., Mückschel, B., Broicher, A., et al. (2015). Applying systems biology tools to study $n$-butanol degradation in Pseudomonas putida KT2440. Eng. Life Sci. 15, 760-771.
Wang, J. C. (2002). Cellular roles of DNA topoisomerases: a molecular perspective. Nat. Rev. Mol. Cell Biol. 3, 430-440. doi: 10.1038/nrm831

Wang, W., Zhou, H., Lin, H., Roy, S., Shaler, T. A., Hill, L. R., et al. (2003). Quantification of Proteins and Metabolites by Mass Spectrometry without Isotopic Labeling or Spiked Standards. Anal. Chem. 75, 4818-4826. doi: 10. 1021/Ac026468x

Ward, P. G., Goff, M., Donner, M., Kaminsky, W., and O'Connor, K. E. (2006). A two step chemo-biotechnological conversion of polystyrene to a biodegradable thermoplastic. Environ. Sci. Technol. 40, 2433-2437. doi: 10.1021/es051 7668

Wehrmann, M., Billard, P., Martin-Meriadec, A., Zegeye, A., and Klebensberger, J. (2017). Functional Role of Lanthanides in Enzymatic Activity and Transcriptional Regulation of Pyrroloquinoline Quinone-Dependent Alcohol Dehydrogenases in Pseudomonas putida KT2440. mBio 8:e00570-17. doi: 10. 1128/mBio.00570-17

Wierckx, N., Koopman, F., Ruijssenaars, H. J., and de Winde, J. H. (2011). Microbial degradation of furanic compounds: biochemistry, genetics, and impact. Appl. Microbiol. Biotechnol. 92, 1095-1105. doi: 10.1007/s00253-0113632-5

Wierckx, N., Prieto, M. A., Pomposiello, P., Lorenzo, V., de, O., Connor, K., et al. (2015). Plastic waste as a novel substrate for industrial biotechnology. Microbiol. Biotechnol. 8, 900-903. doi: 10.1111/1751-7915. 12312

Wilkes, R. A., and Aristilde, L. (2017). Degradation and metabolism of synthetic plastics and associated products by Pseudomonas sp. capabilities and challenges. J. Appl. Microbiol. 123, 582-593. doi: 10.1111/jam.13472

Wynands, B., Lenzen, C., Otto, M., Koch, F., Blank, L. M., and Wierckx, N. (2018). Metabolic engineering of Pseudomonas taiwanensis VLB120 with minimal genomic modifications for high-yield phenol production. Metab. Eng. 47, 121-133. doi: 10.1016/j.ymben.2018.03.011

Yim, H., Haselbeck, R., Niu, W., Pujol-Baxley, C., Burgard, A., Boldt, J., et al. (2011). Metabolic engineering of Escherichia coli for direct production of 1,4-butanediol. Nat. Chem. Biol. 7, 445-452. doi: 10.1038/nchembio.580

Zhou, S., Mohan Raj, S., Ashok, S., Edwardraja, S., Lee, S.-G., and Park, S. (2013). Cloning, expression and characterization of 3-hydroxyisobutyrate dehydrogenase from Pseudomonas denitrificans ATCC 13867. PLoS One 8:e62666. doi: 10.1371/journal.pone.0062666

Zia, K. M., Bhatti, H. N., and Ahmad Bhatti, I. (2007). Methods for polyurethane and polyurethane composites, recycling and recovery: a review. React. Funct. Polym. 67, 675-692. doi: 10.1016/j.reactfunctpolym.2007.05.004

Conflict of Interest: SK was employed by the company Bioplastech Ltd. KO'C was employed by university college Dublin. He has a shareholding in the company Bioplastech Ltd.

The remaining authors declare that the research was conducted in the absence of any commercial or financial relationships that could be construed as a potential conflict of interest.

Copyright (c) 2020 Li, Narancic, Kenny, Niehoff, O'Connor, Blank and Wierckx. This is an open-access article distributed under the terms of the Creative Commons Attribution License (CC BY). The use, distribution or reproduction in other forums is permitted, provided the original author(s) and the copyright owner(s) are credited and that the original publication in this journal is cited, in accordance with accepted academic practice. No use, distribution or reproduction is permitted which does not comply with these terms. 